manuscript No.

(will be inserted by the editor)

\title{
Selected applications of linear semi-infinite systems theory
}

\author{
Miguel A. Goberna ${ }^{\star}$, Andrea B. Ridolfi ${ }^{\star}$, Virginia N. Vera de \\ Serio $^{\star \star \star}$
}

The date of receipt and acceptance will be inserted by the editor

To Marco López in occasion of his 70th anniversary

\begin{abstract}
In this paper we, firstly, review the main known results on systems of an arbitrary (possibly infinite) number of weak linear inequalities posed in the Euclidean space $\mathbb{R}^{n}$ (i.e., with $n$ unknowns), and, secondly, show the potential power of this theoretical tool by developing in detail two significant applications, one to computational geometry: the Voronoi cells, and the other to mathematical analysis: approximate subdifferentials, recovering known results in both fields and proving new ones. In particular, this paper completes the existing theory of farthest Voronoi cells of infinite sets of sites by appealing to well-known results on linear semi-infinite systems.
\end{abstract}

Key words Linear inequality systems - Computational geometry - Voronoi cells $-\varepsilon-$ subdifferentials.

Mathematics Subject Classification (2010) $15 \mathrm{~A} 39,68 \mathrm{U} 05$, 51M20

\section{Introduction}

Linear systems theory is a powerful theoretical tool which has been intensively applied up to now in the semi-infinite programming framework. In recent times, new applications to other fields have emerged, e.g., robust

\footnotetext{
* Department of Mathematics, University of Alicante, Spain. Email: mgoberna@ua.es. Corresponding author.

** CONICET. Universidad Nacional de Cuyo. Facultad de Ciencias Aplicadas a la Industria. Facultad de Ciencias Económicas. Mendoza, Argentina. Email: aridolfi@fcai.uncu.edu.ar

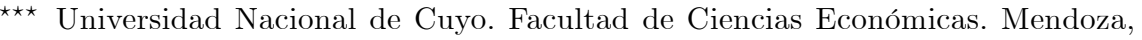
Argentina. Email: virginia.vera@fce.uncu.edu.ar
} 
linear and conic programming, polarity or moment problems. The aim of this paper is, firstly, to review the main known results on systems of an arbitrary (possibly infinite) number of weak linear inequalities posed in the Euclidean space $\mathbb{R}^{n}$, i.e., the so-called linear semi-infinite systems (LSIS in brief), and, secondly, to show the potential power of this theoretical tool by developing in detail two significant applications, one to computational geometry: the Voronoi cells, and the other to approximate subdifferentials. We now briefly describe the main contributions to these research fields and the link between them.

During the 1960s, Charnes, Cooper and Kortanek ([19], [20], [21]) extended the linear programming duality theory to linear optimization problems posed in $\mathbb{R}^{n}$ with infinitely many constraints, calling linear semi-infinite programming (LSIP) this type of optimization problems, without paying special attention to their constraint systems. The first known results on LSIS were the finite dimensional versions of the existence theorem and the non-homogeneous Farkas lemma proved in 1965 by a Chinese mathematician whose family name has been transcribed as Chu (by MathScinet) or as Zhu (by ZentralblatMath). An English version of that work was published in 1966 [22]. Later, Ky Fan gave a variant of the existence theorem [32]. The next two papers on LSIS theory were published by Ulrich Eckhardt, who gave dimension formulas for the solution set [28] and analyzed redundancy phenomena for LSIS [29]. Bruno Brosowsky and his collaborators ([8], [35], [9]) analyzed at the beginning of the 1980s the behavior of the solutions set of continuous LSIS under different types of perturbations. These and other theoretical aspects of LSIS were tackled by Marco A. López and his collaborators, among them the authors of this paper, along the 1980s (e.g., [48] and [44], on deterministic LSIS) and the 1990s ([45], [49], [50], extending to arbitrary LSIS the existing stability theory). The chapters devoted to LSIS in the monograph [46] on LSIP describe the state-of-the-art on LSIS at the end of the 20th Century. The few papers on deterministic LSIS published since 1998 deal with the representation of closed convex sets by means of particular types of LSIS ([1], [40], [60], [37]), while the many contributions to uncertain LSIS in this period cover a variety of topics, as the continuity properties of the solution set and associated maps ([14], [51], [42], [43]), formulas involving error bounds and different types of distances and Lipschitz-like moduli ([13], [10], [15], [12], [11], [16], [17], [18], [61]), as well as radius of robust feasibility [38].

Regarding numerical methods for LSIS, it is worth mentioning the linear semi-infinite feasibility problem, which consists in finding a solution of a given LSIS (see [34] and references therein). Such kind of problem arises in practice in statistics [4], when one tries to solve a given linear or quadratic semi-infinite optimization problem by means of some feasible direction method (see, e.g., [47] and [26], respectively), in variational inequalities [33] and in image recovery problems [23]. Purification methods, in turn, provide an extreme point of the solution set of a given LSIS from any solution (i.e., they are phase I for primal LSIP simplex methods); there 
exist purification methods for particular types of LSIS, analytic and LOP, to be defined in Section 2 ([3], [40], [2]).

The recent survey [47] updates the known connections between LSIP and LSIS theories, and sketches some applications of both disciplines, in the case of LSIS to the computation of the radii of robust feasibility for uncertain linear and conic optimization problems, to polarity of closed convex sets [60] and to moment problems [66], [64], among others. Generally speaking, these applications use the LSIS machinery to prove some results in an easy way, but they do not exploit this tool systematically.

The first published book on computational geometry, due to Preparata and Shamos [65], popularized the nearest Voronoi cells (usually called Voronoi cells) in $\mathbb{R}^{n}$, first used by Descartes (for $n=2$ ), Dirichlet (for $n=3$ ) and Voronoi (for any $n \in \mathbb{N}$ ), and introduced the farthest Voronoi cells, in both cases for finite sets of sites in $\mathbb{R}^{n}, n \in \mathbb{N}$. These concepts have been typically treated via constructive geometry, using ruler and compass when $n=2$ and intersections of spheres and affine manifolds when $n \geq 3$. Voronoi's student Delaunay introduced in 1934 [27] Voronoi cells of infinite discrete sets in the framework of crystallography by using the same tools. The first paper to use an LSIS argument to prove a result was [70], while [56], [57] and [55] systematically applied the theory and methods of linear inequality systems to study nearest Voronoi cells for arbitrary set of sites. The case of farthest Voronoi cells of infinite sets of fixed sites is discussed in [54], where almost all proofs are based on convex analysis arguments.

The subdifferential of a convex function was introduced by Jean-Jacques Moreau and Ralph T. Rockafellar in 1963 ([63], [68]) and the $\varepsilon$-subdifferential (under the name of "approximate subgradients") by Arne Brøndsted and Rockafellar in 1965 [7]. Subdifferentials and $\varepsilon$-subdifferentials are basic tools in the famous textbooks on convex analysis and convex optimization due to Rockafellar [69] and to Jean-Baptiste Hiriart-Urruty and Claude Lemarechal [59], respectively. A recent work by G. Beer, M. J. Cánovas, M. A. López, and J. Parra presents a linear semi-infinite approach to uncertain subdifferentials ([5, Section 2.3]). They revise the stability of the subdifferential for general finite valued convex functions from the LSIS point of view and, in particular, they analyze the calmness property of these subdifferentials.

The aim of this paper is to show that linear inequality systems theory is a powerful instrument in order to obtain geometric information on deterministic Voronoi cells in $\mathbb{R}^{n}, n \in \mathbb{N}$, when the set of sites is infinite and to analyze robustness and stability issues regarding uncertain Voronoi cells of finitely many sites. As far as we know, there is no antecedent on uncertain farthest Voronoi cells of arbitrary sets. Furthermore, we also show that the main known properties about $\varepsilon$-subdifferentials of convex functions can be obtained easily from the LSIS theory, and that we can extend some of these properties for not necessarily convex functions as well.

The paper is organized as follows. Section 2 introduces the necessary notation and provides a brief review on LSIS theory in order to make the paper self-contained and help the researchers to develop future applications 
of this useful theory. The next two sections are devoted to the study of nearest and farthest Voronoi cells when the sites remain fixed (Sections 3) and when the sites are subject to perturbations (Section 4), respectively. For comparative purposes, we study both types of Voronoi cells in parallel, giving simpler proofs of known results together with new ones. The study of the theory of $\varepsilon$-subdifferentials is addressed in Section 5, considering some general functions, not only convex ones. Finally, Section 6 provides the conclusions.

\section{Preliminaries on LSIS theory}

We first introduce the necessary notation. Recall that we focus our study in the Euclidean space $\mathbb{R}^{n}$. Here the scalar product of any $x, y \in \mathbb{R}^{n}$ is designated by $x^{\prime} y$, the Euclidean norm of $x$ by $\|x\|:=\sqrt{x^{\prime} x}$, the Euclidean distance between $x$ and $y$ by $d(x, y)=\|y-x\|$, the zero vector by $0_{n}$, and the closed unit ball by $B_{n}$. For any subset $X \neq \emptyset$, we use the notation span $X$, aff $X$, conv $X$, cone $X, X^{\circ}:=\left\{z \in \mathbb{R}^{n}: x^{\prime} z \leq 0 \forall x \in X\right\}$ and $X^{\perp}:=\left\{z \in \mathbb{R}^{n}: x^{\prime} z=0 \forall x \in X\right\}$ for the linear span of $X$, the affine manifold spanned by $X$, the convex hull of $X$, the convex conical hull of $X \cup\left\{0_{n}\right\}$, the polar of $X$, and the orthogonal subspace to $X$, respectively. We also denote by $\operatorname{int} X, \operatorname{cl} X, \operatorname{bd} X, \operatorname{rbd} X$ and $\operatorname{rint} X$ the interior, the closure, the boundary, the relative boundary, and the relative interior of $X$ (relative means with respect to the topology of aff $X$ ). Finally, $\operatorname{dim} X$ stands for the dimension of a convex set $X$ (i.e., the dimension of aff $X$ ) and $\operatorname{lin} X$ for the lineality space of a convex cone $X$ (i.e., the largest linear subspace contained in $X$ ).

If $X$ is a convex set, $0^{+} X$ denotes the recession cone, and

$$
N_{X}(a):=\left\{y \in \mathbb{R}^{n}:(x-a)^{\prime} y \leq 0 \text { for all } x \in X\right\}
$$

is the normal cone to $X$ at $x$, respectively. It is easy to see that $N_{X}(a)$ is a linear subspace of $\mathbb{R}^{n}$ whenever $a \in \operatorname{rint} X$.

The two basic results in LSIS with fixed data are the finite dimensional versions of the existence theorem and the non-homogeneous Farkas lemma. Direct proofs of these results in a finite dimensional setting were given in [48].

Theorem 1 (Existence theorem) ([22, Theorem 1], [32, Theorem 1], [46, Corollary 3.1.1]) Let $S$ be the solution set of the system $\sigma=\left\{a_{t}^{\prime} x \leq b_{t}, t \in T\right\}$. Then

$$
\begin{aligned}
S \neq \emptyset \Leftrightarrow & \left(\begin{array}{c}
0_{n} \\
-1
\end{array}\right) \notin \operatorname{clcone}\left\{\left(\begin{array}{c}
a_{t} \\
b_{t}
\end{array}\right), t \in T ;\left(\begin{array}{c}
0_{n} \\
1
\end{array}\right)\right\} \\
& \Leftrightarrow\left(\begin{array}{c}
0_{n} \\
-1
\end{array}\right) \notin \operatorname{clcone}\left\{\left(\begin{array}{c}
a_{t} \\
b_{t}
\end{array}\right), t \in T\right\} .
\end{aligned}
$$


Theorem 2 (Non-homogeneous Farkas lemma) ([22, Theorem 2], [46, Corollary 3.1.2]) Let $S \neq \emptyset$ be the solution set of the system $\sigma=\left\{a_{t}^{\prime} x \leq b_{t}, t \in T\right\}$ and let $\left(\begin{array}{l}a \\ b\end{array}\right) \in \mathbb{R}^{n+1}$. Then,

$$
\begin{gathered}
S \subset\left\{x \in \mathbb{R}^{n}: a^{\prime} x \leq b\right\} \\
\Leftrightarrow\left(\begin{array}{l}
a \\
b
\end{array}\right) \in \operatorname{clcone}\left\{\left(\begin{array}{c}
a_{t} \\
b_{t}
\end{array}\right), t \in T ;\left(\begin{array}{c}
0_{n} \\
1
\end{array}\right)\right\} .
\end{gathered}
$$

When $S \neq \emptyset, \sigma$ is said to be consistent. In that case, if $S \subset\left\{x \in \mathbb{R}^{n}: a^{\prime} x \leq b\right\}$, we say that $a^{\prime} x \leq b$ is a linear consequence of $\sigma=\left\{a_{t}^{\prime} x \leq b_{t}, t \in T\right\}$. So, Theorem 2 characterizes the linear consequences of a consistent system $\sigma$. In the infinite dimensional version of Theorems 1 and 2 , the solution set $S$ is contained in a locally convex space $X$ with topological dual $X^{*}$ while the cones are contained in the product space $X^{*} \times \mathbb{R}$. For this reason, we can interpret the above theorems as providing dual characterizations of consistency and linear consequences, respectively.

The reference cone of a nonempty closed convex set $X$ is defined as

$$
K(X)=\left\{(a, b) \in \mathbb{R}^{n+1}: a^{\prime} x \leq b \text { for all } x \in X\right\},
$$

So, Theorem 2 can be reformulated as asserting that, if $X \neq \emptyset$ is the solution set of $\left\{a_{t}^{\prime} x \leq b_{t}, t \in T\right\}$, then

$$
K(X)=\operatorname{clcone}\left\{\left(\begin{array}{c}
a_{t} \\
b_{t}
\end{array}\right), t \in T ;\left(\begin{array}{c}
0_{n} \\
1
\end{array}\right)\right\} .
$$

which shows that $K(X)$ is a closed convex cone.

The following result can be straightforwardly derived from the above implication:

Corollary 1 (Dual characterization of the inclusion) Given two nonempty closed convex sets $X, Y \subset \mathbb{R}^{n}, X \subset Y$ if and only if $K(Y) \subset K(X)$. Hence $Y=X$ if and only if $K(Y)=K(X)$.

The next four results also follow from Theorem 2 . The first one characterizes the homogeneous inequalities which are consequence of an homogeneous system.

Corollary 2 (Homogeneous Farkas lemma) Let $S_{0}$ be the solution set of the homogeneous system $\sigma_{0}=\left\{a_{t}^{\prime} x \leq 0, t \in T\right\}$ and let $a \in \mathbb{R}^{n}$. Then,

$$
S_{0} \subset\left\{x \in \mathbb{R}^{n}: a^{\prime} x \leq 0\right\} \Leftrightarrow a \in \operatorname{cl} \text { cone }\left\{a_{t}, t \in T\right\} .
$$

A well-known consequence of Corollary 2 is that $K^{\circ \circ}=\operatorname{cl} K$ for any convex cone $K$. 
Corollary 3 (Boundedness) [46, Theorem 9.3] Let $S$ be the solution set of the consistent system $\sigma=\left\{a_{t}^{\prime} x \leq b_{t}, t \in T\right\}$, with reference cone $K(S)$. Then the following statements are equivalent:

(i) $S$ is bounded.

(ii) The unique solution of $\left\{a_{t}^{\prime} x \leq 0, t \in T\right\}$ is $0_{n}$, i.e., $0^{+} S=\left\{0_{n}\right\}$.

(iii) $\left(\begin{array}{c}0_{n} \\ 1\end{array}\right) \in \operatorname{int} K(S)$.

(iv) cone $\left\{a_{t}, t \in T\right\}=\mathbb{R}^{n}$.

We now state a duality formula for $\operatorname{dim} S$.

Corollary 4 (Dimension) [46, Theorem 5.8] Let $S$ be a nonempty closed convex set with reference cone $K(S)$. Then

$$
\operatorname{dim} S=n-\operatorname{dim} \operatorname{lin} K(S) .
$$

The next result gathers together characterizations, through the properties of the corresponding reference cones, of 11 classes of closed convex sets, some of them playing important roles in optimization and geometry, as the polyhedral convex sets (polyhedra in brief, which are the feasible and optimal sets in linear programming), the sums of compact convex sets with closed convex cones (called Motzkin decomposable in [36], whose corresponding functions, also called Motzkin decomposable, attain their minima when they are bounded from below [53]), the sums of compact convex sets with linear subspaces (the typical structure of the optimal set in convex programming, see [52] and references therein, subfamily of Motzkin decomposable sets without specific name to the best of our knowledge), the $k$-simplices (the basic tool of the celebrated Nelder and Mead Method for optimization without derivatives), the sums of $n$ segments whose directions form a basis of $\mathbb{R}^{n}$ (called Voronoi parallelotopes, or just parallelotopes, in [24], whose volumes can be computed via Gram determinants), the convex hulls of the union of a pair of parallel affine manifolds of dimension $k-1$ (called $k$-sandwiches, which were studied in [41] in the framework of illumination of convex bodies), etc. Lemma 1 below characterizes two other types of closed convex sets.

Corollary 5 (Dual characterizations of some families of sets) ([46, Theorem 5.13], [41]) Let $S$ be a nonempty closed convex set with reference cone $K(S)$. Then the following statements hold:

(i) $S$ is a singleton $\Leftrightarrow K(S)$ is a halfspace.

(ii) $S$ is a polyhedron $\Leftrightarrow K(S)$ is a polyhedron.

(iii) $S$ is an affine manifold $\Leftrightarrow K(S) \cap(\operatorname{lin} K(S))^{\perp}=\operatorname{cone}\left\{\left(0_{n}, 1\right)\right\}$.

(iv) $S$ is full-dimensional $\Leftrightarrow K(S)$ is pointed.

(v) $S$ is compact $\Leftrightarrow\left(0_{n}, 1\right) \in \operatorname{int} K(S)$.

(vi) $S$ is a polytope $\Leftrightarrow K(S)$ is a polyhedron and $\left(0_{n}, 1\right) \in \operatorname{int} K(S)$.

(vii) $S$ is the sum of a compact convex set with a linear subspace $\Leftrightarrow\left(0_{n}, 1\right) \in$ $\operatorname{rint} K(S)$. 
(viii) $S$ is Motzkin decomposable $\Leftrightarrow$ There exist two closed convex cones $C \subset \mathbb{R}^{n+1}$ and $L \subset \mathbb{R}^{n}$ such that $K(S)=C \cap(L \times \mathbb{R}),\left(0_{n},-1\right) \notin C$ and $\left(0_{n}, 1\right) \in \operatorname{int} C$.

(ix) $S$ is a $k$-simplex $\Leftrightarrow\left(0_{n}, 1\right) \in \operatorname{int} K(S), \operatorname{dim} \operatorname{lin} K(S)=n-k$, and the pointed cone of $K(S)$ has $k+1$ extreme rays.

(x) $S$ is a $k$-sandwich $\Leftrightarrow K(S)$ is $K(S)=C+W$, where $C$ is a pointed closed convex cone and $W$ is a linear subspace such that $\operatorname{dim} C=2, \operatorname{dim} W=$ $n-k,\left(0_{n}, 1\right) \in \operatorname{rint} C$, and $C \cap\left(W+\operatorname{span}\left\{\left(0_{n}, 1\right)\right\}\right)=\operatorname{cone}\left\{\left(0_{n}, 1\right)\right\}$.

(xi) $S$ is a parallelotope $\Leftrightarrow K(S)=\operatorname{cone}\left\{\left(\begin{array}{c}a_{i} \\ \alpha_{i}\end{array}\right), i \in I ;-\left(\begin{array}{c}a_{i} \\ \beta_{i}\end{array}\right), i \in I ;\left(\begin{array}{c}0_{n} \\ 1\end{array}\right)\right\}$, with $I=\{1, \ldots, n\},\left\{a_{i}, i \in I\right\}$ linearly independent, and $\alpha_{i}<\beta_{i}$ for $i \in I$.

In the following we make also use of two types of closed convex sets which do not belong to the families listed in the previous corollary, one of them are the coradiant sets and the other one are the pointed convex cones. Recall that a nonempty set $C$ is said to be coradiant with respect to $s \in \mathbb{R}^{n}$ if, whenever $x \in C$ and $\lambda \geq 1$, then $(1-\lambda) s+\lambda x \in C$. Examples of coradiant sets are the translates of cones by the vector $s$, that we call cones with apex $s$. Obviously, any cone with apex $s \in \mathbb{R}^{n}$ is coradiant with respect to (w.r.t. in brief) $s$ while the converse is not true (for instance, $C:=\left\{x \in \mathbb{R}^{n}:\|x\| \geq 1\right\}$ is coradiant w.r.t. $0_{n}$, but it is not a cone with apex $\left.0_{n}\right)$.

Lemma 1 (Dual characterizations of coradiant sets and cones) Let $S \neq \emptyset$ be the solution set of $\sigma=\left\{a_{t}^{\prime} x \leq b_{t}, t \in T\right\}$ and $s \in \mathbb{R}^{n}$. Then, the following statements hold true:

(i) $S$ is coradiant w.r.t. $s$ if and only if

$$
\left(\begin{array}{c}
a_{t} \\
0
\end{array}\right) \in \mathrm{cl} \text { cone }\left\{\left(\begin{array}{c}
a_{t} \\
b_{t}-a_{t}^{\prime} s
\end{array}\right), t \in T,\left(\begin{array}{c}
0_{n} \\
1
\end{array}\right)\right\}, \forall t \in T .
$$

Moreover, if

$$
a_{t}^{\prime} s \geq b_{t}, \forall t \in T,
$$

then $S$ is coradiant w.r.t. $s$, and the converse holds whenever $T$ is finite and each ray $\mathbb{R}_{+} a_{t}$ defines a different edge of cone $\left\{a_{t}, t \in T\right\}, t \in T$.

(ii) $S$ is a cone with apex at $s$ if and only if

$$
\text { cl cone }\left\{\left(\begin{array}{c}
a_{t} \\
b_{t}-a_{t}^{\prime} s
\end{array}\right), t \in T,\left(\begin{array}{c}
0_{n} \\
1
\end{array}\right)\right\}=\operatorname{cl} \text { cone }\left\{a_{t}, t \in T\right\} \times \mathbb{R}_{+} .
$$

A sufficient condition is that $a_{t}^{\prime} s=b_{t}$ for all $t \in T$, and the converse holds under the same conditions as in (i).

Proof. Let $K:=$ cone $\left\{\left(\begin{array}{c}a_{t} \\ b_{t}-a_{t}^{\prime} s\end{array}\right), t \in T,\left(\begin{array}{c}0_{n} \\ 1\end{array}\right)\right\}$ along the proof.

(i) It is easy to see that the convex solution set $S$ is coradiant w.r.t. $0_{n}$ if and only if $S \subset 0^{+} S$. So, $S$ is coradiant w.r.t. $s$ if and only if $S-$ 
$s \subset 0^{+}(S-s)=0^{+} S$. Since $S-s=\left\{x: \mathbb{R}^{n}: a_{t}^{\prime}(x+s) \leq b_{t}, t \in T\right\}$ and $0^{+} S=\left\{x: \mathbb{R}^{n}: a_{t}^{\prime} x \leq 0, t \in T\right\}$, by Corollary $1, S$ is coradiant w.r.t. $s$ if and only if

$$
\text { cl cone }\left\{a_{t}, t \in T\right\} \times \mathbb{R}_{+} \subset \operatorname{cl} K,
$$

if and only if (2) holds.

Assuming (3), one has

$$
\left(\begin{array}{c}
a_{t} \\
0
\end{array}\right)=\left(\begin{array}{c}
a_{t} \\
b_{t}-a_{t}^{\prime} s
\end{array}\right)+\left(a_{t}^{\prime} s-b_{t}\right)\left(\begin{array}{c}
0_{n} \\
1
\end{array}\right), \forall t \in T,
$$

so that (2) holds.

We now assume that (2) holds, $T$ is finite and each ray $\mathbb{R}_{+} a_{t}$ defines a different edge of cone $\left\{a_{t}, t \in T\right\}, t \in T$. The finiteness assumption entails the closedness of $K$ and of cone $\left\{a_{t}, t \in T\right\}$. Fixing $t \in T$, there is a unique way of writing $a_{t}$ as nonnegative linear combination of the vectors of $\left\{a_{t}, t \in T\right\}, a_{t}=1 a_{t}$, so that (2) implies the existence of some nonnegative scalar $\lambda_{t}$ such that

$$
\left(\begin{array}{c}
a_{t} \\
0
\end{array}\right)=\left(\begin{array}{c}
a_{t} \\
b_{t}-a_{t}^{\prime} s
\end{array}\right)+\lambda_{t}\left(\begin{array}{c}
0_{n} \\
1
\end{array}\right)
$$

which entails $b_{t}-a_{t}^{\prime} s=-\lambda_{t} \leq 0$.

(ii) $S$ is a cone with apex $s$ if and only if $S-s=0^{+}(S-s)=0^{+} S$, if and only if (4) holds. The rest of the proof is as the one of (i).

To go further with the obtaining of information on $S$ from the data (the coefficients of $\sigma$ ), one needs some data qualifications (conditions involving the coefficients of the system).

A vector $\widehat{x} \in \mathbb{R}^{n}$ is called a Slater point of $\sigma=\left\{a_{t}^{\prime} x \leq b_{t}, t \in T\right\}$ if $a_{t}^{\prime} \widehat{x}<$ $b_{t}$ for all $t \in T$, in which case we say that $\sigma$ satisfies the Slater condition. Following [67], we say that a consistent system $\sigma=\left\{a_{t}^{\prime} x \leq b_{t}, t \in T\right\}$ is locally Farkas-Minkowski (LFM in brief) if every linear consequence of $\sigma$ determining a supporting hyperplane to $S$ is also the consequence of a finite subsystem of $\sigma$. Sufficient conditions for a system $\sigma=\left\{a_{t}^{\prime} x \leq b_{t}, t \in T\right\}$ to be LFM is the closedness of cone $\left\{\left(\begin{array}{c}a_{t} \\ b_{t}\end{array}\right), t \in T ;\left(\begin{array}{c}0_{n} \\ 1\end{array}\right)\right\}$, which holds whenever $\sigma$ satisfies the Slater condition and it is continuous (i.e., $T$ is compact and the function $t \longmapsto\left(a_{t}, b_{t}\right)$ is continuous on $\left.T\right)$. In particular, any finite system is LFM.

Given a consistent system $\sigma=\left\{a_{t}^{\prime} x \leq b_{t}, t \in T\right\}$ with solution set $S$, we say that an index $t \in T$ is carrier whenever $a_{t}^{\prime} x=b_{t}$ for all $x \in S$. We denote by $T^{=}:=\left\{t \in T: a_{t}^{\prime} x=b_{t}, \forall x \in S\right\}$ the set of carrier indices of $\sigma$. The exposed face of $S$ associated with index $t \in T$ is the closed convex set $S_{t}:=\left\{x \in S: a_{t}^{\prime} x=b_{t}\right\}$.

Theorem 3 (Geometry of the solution set) [67] Let $\sigma=\left\{a_{t}^{\prime} x \leq b_{t}, t \in T\right\}$ be LFM with solution set $S$. Then the following statements hold: 
(i) $\operatorname{dim} S=n-\operatorname{dim} \operatorname{span}\left\{a_{t}, t \in T^{=}\right\}$.

(ii) $\operatorname{rbd} S=\cup\left\{S_{t}: t \in T \backslash T^{=}\right\}$.

(iii) bd $S=\cup\left\{S_{t}: a_{t} \neq 0_{n}, t \in T\right\}$.

In particular, if $T^{=}=\emptyset, \operatorname{dim} S=n$ and

$$
\operatorname{rint} S=\operatorname{int} S=\left\{x \in \mathbb{R}^{n}: a_{t}^{\prime} x<b_{t}, t \in T\right\}
$$

is the set of Slater points of $\sigma$.

Given $x \in S, A(x):=$ cone $\left\{a_{t}: a_{t}^{\prime} x=b_{t}, t \in T\right\}$ is the so-called $a c$ tive cone at $x$, which plays a crucial role in LSIP. Following [1], $\sigma=$ $\left\{a_{t}^{\prime} x \leq b_{t}, t \in T\right\}$ is called locally polyhedral (LOP in short) whenever $A(x)^{\circ}$ is the cone of feasible directions to $S$ at $x$ for all $x \in S$, and, following [3], it is called analytic whenever $T$ is a compact one-dimensional interval and the $n+1$ components of the function $t \longmapsto\left(a_{t}, b_{t}\right)$ are analytic in the variable $t$. If $\sigma$ is LOP, then its solution set is a quasipolyhedron (i.e., its nonempty intersections with polytopes are polytopes).

The next result characterizes the faces of $S$ in terms of a linear subspace $L(x)$ associated with each $x \in S$ which is defined differently for LOP and analytic systems:

- If $\sigma$ is LOP, $L(x):=\operatorname{span}\left\{a_{t}: a_{t}^{\prime} x=b_{t}, t \in T\right\}$.

- If $\sigma$ is analytic, $L(x):=\operatorname{span}\left\{a_{t}, t \in T\right\}$ if $a_{t}^{\prime} x=b_{t}$ for all $t \in T$; otherwise, $L(x)$ is the linear span of the union of the sets of successive derivatives $\left\{a_{t}, a_{t}^{(1)}, \ldots, a_{t}^{(d(t))}\right\}$ at those indices $t \in T$ which are roots (with order of multiplicity $d(t)+1$ ) of the slack function at $x, t \longmapsto$ $a_{t}^{\prime} x-b_{t}$.

Theorem 4 (Facial structure of the solution set) [40, Corollary 2.1] Let $\sigma$ be a LOP or an analytic linear representation of $S$, and let $G$ be a nonempty closed convex subset of $S$ such that $\operatorname{dim} G=k$. Then, $G$ is a face of $S$ if and only if $\operatorname{dim} L(x)=n-k$ for certain (every) $x \in \operatorname{rint} G$ and $\operatorname{dim} L(x)>n-k$ for every $x \in \operatorname{rbd} G$.

This result allows to obtain characterizations of the extreme points and the edges of $S$.

Corollary 6 (Extreme points and edges) ([1, THeorems 9.1 and 9.2], [3], [40]) If $\sigma$ is either LOP or analytic with solution set $S$, then the following statements hold:

(i) $x \in S$ is an extreme point of $S$ if and only if $L(x)=\mathbb{R}^{n}$;

(ii) $\left[x^{1}, x^{2}\right]$ is an edge of $S$ if and only if $x^{1}$ and $x^{2}$ are extreme points of $S$ and $\operatorname{dim} L(x)=n-1$ for a certain (every) $x \in] x^{1}, x^{2}[$;

(iii) $\{x+\mu y: \mu \geq 0\}$ is an edge of $S$ if and only if $x$ is an extreme point of $S$ and $\operatorname{dim} L(x+\mu y)=n-1$ for certain (every) $\mu>0$;

(iv) $\{x+\mu y: \mu \in \mathbb{R}\}$ is an edge of $S$ if and only if $x \in S, \pm y \in 0^{+} S$ and $\operatorname{dim} L(x+\mu y)=n-1$ for certain (every) $\mu \in \mathbb{R}$. 
We now suppose that not all data of the given LSIS, the so-called nominal system $\bar{\sigma}=\left\{\bar{a}_{t}^{\prime} x \leq \bar{b}_{t}, t \in T\right\}$, are fixed. In that case, we say that we are dealing with an uncertain system $\sigma=\left\{a_{t}^{\prime} x \leq b_{t}, t \in T\right\}$ whose coefficients are the result of perturbing those of $\bar{\sigma}$. We denote by $\bar{S}$ and $S$ the solution sets of $\bar{\sigma}$ and $\sigma$, which are deterministic and uncertain sets, respectively. Uncertain systems can be approached from different perspectives, e.g., the robust and the parametric ones.

The robust approach associates to $\bar{\sigma}$, assumed to be consistent, a robust counterpart system $\sigma_{R}$ consisting in the aggregation to $\bar{\sigma}$ of all admissible perturbed constraints (see, e.g., [6]). We denote by $S_{R}$ the solution set of $\sigma_{R}$, called robust solution set. If we admit too many perturbed constraints, $\sigma_{R}$ is no longer consistent. So, the main objective of this approach is to force the consistency of $\sigma_{R}$ by controlling the admissible perturbations.

Assume that, for each $t \in T$, the admissible perturbations of $\bar{a}_{t}^{\prime} x \leq \bar{b}_{t}$ have the form $a^{\prime} x \leq b$, with $(a, b) \in \mathcal{U}_{t}=\left(\bar{a}_{t}, \bar{b}_{t}\right)+r_{t} B_{n+1}$, with $r_{t} \in \mathbb{R}_{+}$. Since the uncertainty sets $\mathcal{U}_{t}$ are balls, this framework is called ball uncertainty. Obviously, $r_{t}=0$ means that the coefficients of the corresponding inequality $\bar{a}_{t}^{\prime} x \leq \bar{b}_{t}$ are deterministic (this is typically the case of the sign constraints in many decision problems). We associate with each $\alpha \in \mathbb{R}_{+}$the system

$$
\sigma_{R}^{\alpha}:=\left\{a_{t}^{\prime} x \leq b_{t},\left(a_{t}, b_{t}\right) \in\left(\bar{a}_{t}, \bar{b}_{t}\right)+\alpha B_{n+1}, t \in T\right\},
$$

whose correspondent solution set we denote by $S_{R}^{\alpha}$. The radius of feasibility of $\bar{\sigma}$ is defined as

$$
\rho(\bar{\sigma}):=\sup \left\{\alpha \in \mathbb{R}_{+}: S_{R}^{\alpha} \neq \emptyset\right\} .
$$

Since the balls $\left(\bar{a}_{t}, \bar{b}_{t}\right)+\alpha B_{n+1}$ intersect the ray $\mathbb{R}_{+}\left(0_{n},-1\right)$ for $\alpha$ sufficiently large, we have $\rho(\bar{\sigma})<+\infty$ by Theorem 1 . Formulas for $\rho(\bar{\sigma})$ for $T$ arbitrary have been obtained from the LSIS stability theory (e.g., [38, Theorem 2.5]) and for $T$ finite in a direct way [39, Theorem 4]. These formulas involve the so-called epigraphical set (term introduced in [15]):

$$
E(\bar{\sigma}):=\operatorname{conv}\left\{\left(\bar{a}_{t}, \bar{b}_{t}\right), t \in T\right\}+\mathbb{R}_{+}\left\{\left(0_{n}, 1\right)\right\} .
$$

In general, $E(\bar{\sigma})$ is a convex set, but it is a polyhedron whenever $T$ is finite.

Theorem 5 (Radius of feasibility) ([38, Theorem 2.5]) The radius of feasibility for ball uncertainty is

$$
\rho(\bar{\sigma})=d\left(0_{n+1}, E(\bar{\sigma})\right) .
$$

Since $d\left(0_{n+1}, E(\bar{\sigma})\right)$ is nothing else than the minimum value of $\|\cdot\|$ on $E(\bar{\sigma})$, when $T$ is finite $\rho(\bar{\sigma})$ can easily be computed by solving a convex quadratic program. By the very definition of $\rho(\bar{\sigma})$,

$$
\sup _{t \in T} r_{t}<\rho(\bar{\sigma}) \Longrightarrow\left\{x \in \mathbb{R}^{n}: a^{\prime} x \leq b,(a, b) \in \bigcup_{t \in T} \mathcal{U}_{t}\right\} \neq \emptyset
$$


The parametric approach, in the LSIS setting, consists in embedding the nominal system $\bar{\sigma}$ into a space of systems, $\Omega$, representing the results of perturbing the coefficients of $\bar{\sigma}$ but preserving the number of variables and the index set. The elements of $\Omega$, called parameters, are here systems of the form $\sigma=\left\{a_{t}^{\prime} x \leq b_{t}, t \in T\right\}$, also represented as $\sigma=(a ., b$. $)$, with a. $: T \longrightarrow \mathbb{R}^{n}$ and $b$. $: T \longrightarrow \mathbb{R}$ such that $a(t)=a_{t}$ and $b(t)=b_{t}$, $t \in T$, so that $\Omega=\left(\mathbb{R}^{n}\right)^{T} \times \mathbb{R}^{T}$. It is worth observing that, in certain circumstances, the admissible perturbations may affect only part of the coefficients of $\bar{\sigma}$ (e.g., the right-hand side $\bar{b}_{t}$ ) or the coefficients may depend on some parameter (as it happens with the Voronoi cells, which are solution sets of linear systems involving the sites), in which case the parameters are the uncertain elements in $\bar{\sigma}$ (e.g., the uncertain sites in the case of uncertain Voronoi cells).

The solution set map $\mathcal{S}: \Omega \rightrightarrows \mathbb{R}^{n}$ is the multifunction (or set-valued mapping) that associates to each $\sigma \in \Omega$ its solution set $\mathcal{S}(\sigma)=S$. The domain of $\mathcal{S}$ is $\operatorname{dom} \mathcal{S}=\{\sigma \in \Omega: \mathcal{S}(\sigma) \neq \emptyset\}$. A vector $\widehat{x} \in \mathbb{R}^{n}$ is strong Slater point of $\sigma=\left\{a_{t}^{\prime} x \leq b_{t}, t \in T\right\}$ if there exists $\varepsilon>0$ such that $a_{t}^{\prime} \widehat{x} \leq b_{t}-\varepsilon$ for all $t \in T$, in which case we say that $\sigma$ satisfies the strong Slater condition. To analyze the continuity properties of $\mathcal{S}$ we must define a topology on $\Omega$. Since we are assuming here that all coefficients of $\bar{\sigma}$ can be arbitrarily perturbed, we assume that $\Omega$ is equipped with the pseudonorm of the uniform convergence on $T$, e.g.,

$$
\|\sigma-\bar{\sigma}\|_{\infty}=\sup _{t \in T}\left\|\left(\begin{array}{l}
a_{t} \\
b_{t}
\end{array}\right)-\left(\begin{array}{c}
\bar{a}_{t} \\
\bar{b}_{t}
\end{array}\right)\right\| .
$$

Stability of a given system $\bar{\sigma}$ intuitively means that its solution set $\bar{S}$ does not change abruptly under small perturbations of the coefficients (preserving $n$ and $T$ ) producing a perturbed system $\sigma=\left\{a_{t}^{\prime} x \leq b_{t}, t \in T\right\}$ with solution set $S$. For the sake of completeness, we recall now the stability concepts for set-valued mappings introduced by Bouligand and Kuratowski that we shall consider in this paper. Let $\Omega$ and $X$ be two metric spaces and let $\mathcal{A}: \Omega \rightrightarrows X$ be a set-valued mapping, then:

$\mathcal{A}$ is closed at $\bar{\omega} \in \operatorname{dom} \mathcal{A}$ if for all sequences $\left\{\omega_{r}\right\} \subset \Omega$ and $\left\{x_{r}\right\} \subset X$ satisfying $x_{r} \in \mathcal{A}\left(\omega_{r}\right)$ for all $r \in \mathbb{N}, \omega_{r} \rightarrow \bar{\omega}$ and $x_{r} \rightarrow x_{0} \in X$, one has $x_{0} \in \mathcal{A}(\bar{\omega})$.

$\mathcal{A}$ is lower semicontinuous at $\bar{\omega} \in \Omega$ (the nominal parameter) in the Berge sense (lsc, in brief) if, for each open set $W \subset X$ such that $W \cap \mathcal{A}(\bar{\omega}) \neq$ $\emptyset$, there exists an open set $V \subset \Omega$, containing $\bar{\omega}$, such that $W \cap \mathcal{A}(\omega) \neq \emptyset$ for each $\omega \in V$.

$\mathcal{A}$ is upper semicontinuous at $\bar{\omega} \in \Omega$ in the Berge sense (usc, in brief) if, for each open set $W \subset X$ such that $\mathcal{A}(\bar{\omega}) \subset W$, there exists an open set $V \subset \Omega$, containing $\bar{\omega}$, such that $\mathcal{A}(\omega) \subset W$ for each $\omega \in V$.

It is well known that a set-valued mapping $\mathcal{A}$ is usc at a point in its domain whenever it is closed and locally bounded at that point.

Related to the solution set map $\mathcal{S}$, the basic result, for arbitrary perturbations of all the coefficients, involves a parameter space $\Omega$ where $\sigma$ 
moves and the solution set multifunction $\mathcal{S}: \Omega \rightrightarrows \mathbb{R}^{n}$ associating to each system $\sigma$ its corresponding solution set $\mathcal{S}(\sigma)$. There exist many stability concepts, the main ones being equivalent to the lower semicontinuity of $\mathcal{S}$ at $\bar{\sigma}$ according to the next basic result.

Theorem 6 (Lower semicontinuity of the solution set mapping) [46, Theorem 6.1] Let $\bar{\sigma} \in \operatorname{dom} \mathcal{S}$. The following statements are equivalent:

(i) $\mathcal{S}$ is lsc at $\bar{\sigma}$.

(ii) $\bar{\sigma} \in \operatorname{int} \operatorname{dom} \mathcal{S}$, i.e., sufficiently small perturbations of $\bar{\sigma}$ preserve its feasibility.

(iii) $\bar{\sigma}$ satisfies the strong Slater condition.

(iv) $0_{n+1} \notin \operatorname{cl} \operatorname{conv}\left\{\left(\bar{a}_{t}, \bar{b}_{t}\right), t \in T\right\}$.

When $T$ is finite, one can replace "strong Slater" by just "Slater" in (ii) and eliminate "cl" in (iv). Many other equivalent conditions appear in [46, Theorem 6.1], [42] and [43].

Theorem 7 (Upper semicontinuity of the solution set mapping) [46, Corolary 6.2.1] Let $\bar{\sigma} \in \operatorname{dom} \mathcal{S}$. If $\mathcal{S}(\bar{\sigma})$ is bounded, then $\mathcal{S}$ is usc at $\bar{\sigma}$. The converse holds whenever $T$ is finite.

A hardly checkable characterization of the upper semicontinuity of $\mathcal{S}$ for arbitrary index sets was given in [13].

\section{Deterministic Voronoi cells}

The set of Voronoi sites is a given nonempty set $T \subset \mathbb{R}^{n}$. The nearest Voronoi cell, or just Voronoi cell, of the generator $s \in T, V_{T}(s)$, consists of all points in $\mathbb{R}^{n}$ closer to $s$ than to any other site $t \in T$, i.e.,

$$
V_{T}(s)=\left\{x \in \mathbb{R}^{n}:\|x-s\| \leq\|x-t\|, t \in T \backslash\{s\}\right\} .
$$

Analogously, the farthest Voronoi cell of $s \in T, F_{T}(s)$, is the set of all those points in $\mathbb{R}^{n}$ which are farther from the site $s$ than to any other $t \in T$, i.e.,

$$
F_{T}(s)=\left\{x \in \mathbb{R}^{n}:\|x-s\| \geq\|x-t\|, t \in T \backslash\{s\}\right\} .
$$

Both types of cells, (8) and (9), can be expressed as the feasible set of particular linear inequality systems. Indeed, after straightforward calculations, we have

$$
V_{T}(s)=\left\{x \in \mathbb{R}^{n}: 2(t-s)^{\prime} x \leq\|t\|^{2}-\|s\|^{2}, t \in T \backslash\{s\}\right\}
$$

and

$$
F_{T}(s)=\left\{x \in \mathbb{R}^{n}:-2(t-s)^{\prime} x \leq\|s\|^{2}-\|t\|^{2}, t \in T \backslash\{s\}\right\} .
$$

Notice that for an infinite set of sites, e.g., when $T$ is a curve or an infinite discrete set, the systems in (10) and (11) are LSIS. These two systems 
are crucial in obtaining geometric properties of $V_{T}(s)$ and $F_{T}(s)$, such as boundedness, polyhedrality, facial structure, and extreme points and edges.

The reference cones of $V_{T}(s)$ and $F_{T}(s)$ are

$$
K\left(V_{T}(s)\right)=\text { cl cone }\left\{\left(\begin{array}{c}
2(t-s) \\
\|t\|^{2}-\|s\|^{2}
\end{array}\right), t \in T ;\left(\begin{array}{c}
0_{n} \\
1
\end{array}\right)\right\}
$$

and

$$
K\left(F_{T}(s)\right)=\mathrm{cl} \text { cone }\left\{\left(\begin{array}{c}
-2(t-s) \\
\|s\|^{2}-\|t\|^{2}
\end{array}\right), t \in T ;\left(\begin{array}{c}
0_{n} \\
1
\end{array}\right)\right\},
$$

respectively.

The Voronoi cell $V_{T}(s)$ is always nonempty, but it is not the case for the farthest Voronoi cell $F_{T}(s)$. Indeed, [54] employs the linear representation (11) and the existence Theorem 1 for LSIS to establish the following existence theorem for farthest Voronoi cells:

$$
F_{T}(s) \neq \emptyset \Longleftrightarrow\left(\begin{array}{c}
0_{n} \\
-1
\end{array}\right) \notin \text { cl cone }\left\{\left(\begin{array}{c}
-2(t-s) \\
\|s\|^{2}-\|t\|^{2}
\end{array}\right), t \in T\right\} .
$$

From Corollary 4,

$$
\operatorname{dim} V_{T}(s)=n-\operatorname{dim} \operatorname{lin} K\left(V_{T}(s)\right)
$$

and, assuming that $F_{T}(s) \neq \emptyset$,

$$
\operatorname{dim} F_{T}(s)=n-\operatorname{dim} \operatorname{lin} K\left(F_{T}(s)\right) .
$$

The Voronoi diagram and the farthest Voronoi diagram of $T$ are the families of sets $\left\{V_{T}(s), s \in T\right\}$ and $\left\{F_{T}(s), s \in T\right\}$, respectively. If $T$ is a singleton, both diagrams trivially coincide with $\left\{\mathbb{R}^{n}\right\}$. So, we can assume that $T$ contains at least two different sites.

It follows immediately that

$$
V_{T}(s)=s+V_{T-s}\left(0_{n}\right) \text { and } F_{T}(s)=s+F_{T-s}\left(0_{n}\right) .
$$

Thus, it is possible just to assume $s=0_{n}$ and consider the cones

$$
K\left(V_{T}\left(0_{n}\right)\right)=\operatorname{clcone}\left\{\left(\begin{array}{c}
2 t \\
\|t\|^{2}
\end{array}\right), t \in T ;\left(\begin{array}{c}
0_{n} \\
1
\end{array}\right)\right\}
$$

and

$$
K\left(F_{T}\left(0_{n}\right)\right)=\operatorname{clcone}\left\{\left(\begin{array}{c}
-2 t \\
-\|t\|^{2}
\end{array}\right), t \in T ;\left(\begin{array}{c}
0_{n} \\
1
\end{array}\right)\right\},
$$

respectively.

Proposition 1 (Basic properties of cells) Let $T \subset \mathbb{R}^{n}$ and $s \in T$. Then the following statements hold:

(i) $V_{T}(s)$ and $F_{T}(s)$ are closed convex sets.

(ii) If $F_{T}(s) \neq \emptyset$, then $F_{T}(s)$ is coradiant with respect to $s, T$ is bounded and $s$ is an extreme point of $\operatorname{conv} T$. 
Proof. (i) follows from (10) and (11).

(ii) Assume that $F_{T}(s) \neq \emptyset$. We prove the coradiant property of $F_{T}(s)$ appealing to Lemma 1 . Since $F_{T}(s)$ is the solution set of the system

$$
\left\{a_{t}^{\prime} x \leq b_{t}, t \in T \backslash\{s\}\right\},
$$

with $a_{t}=-2(t-s)$ and $b_{t}=\|s\|^{2}-\|t\|^{2}, t \in T \backslash\{s\}$, the sufficient condition (3) holds as

$$
b_{t}-a_{t}^{\prime} s=\|s\|^{2}-\|t\|^{2}+2(t-s)^{\prime} s=-\|t-s\|^{2} \leq 0, \forall t \in T \backslash\{s\} .
$$

We conclude that $F_{T}(s)$ is coradiant w.r.t. $s$.

For the rest of the proof we can assume without loss of generality (w.l.o.g.) that $s=0_{n} \in T$.

If $T$ is unbounded, we can take a sequence $\left\{t_{k}\right\} \subset T$ such that $\left\|t_{k}\right\| \longrightarrow$ $+\infty$. Since $\frac{1}{\left\|t_{k}\right\|^{2}}\left(\begin{array}{c}-2 t_{k} \\ -\left\|t_{k}\right\|^{2}\end{array}\right) \longrightarrow\left(\begin{array}{c}0_{n} \\ -1\end{array}\right)$, it follows that

$$
\left(\begin{array}{c}
0_{n} \\
-1
\end{array}\right) \in \operatorname{clcone}\left\{\left(\begin{array}{c}
-2 t \\
-\|t\|^{2}
\end{array}\right), t \in T\right\}
$$

and $F_{T}(s)=\emptyset$ by $(12)$. So, $F_{T}(s) \neq \emptyset$ entails the boundedness of $T$.

If $0_{n}$ is not an extreme point of $\operatorname{conv} T$, then there exist $t_{1}, \ldots, t_{m} \in$ $T \backslash\left\{0_{n}\right\}$ and $\lambda_{1}, \ldots, \lambda_{m}>0$ such that $\sum_{i=1}^{m} \lambda_{i} t_{i}=0_{n}$ and $\sum_{i=1}^{m} \lambda_{i}=1$. Then

$$
\left(\begin{array}{c}
0_{n} \\
-\sum_{i=1}^{m} \lambda_{i}\left\|t_{i}\right\|^{2}
\end{array}\right)=\sum_{i=1}^{m} \lambda_{i}\left(\begin{array}{c}
-2 t_{i} \\
-\left\|t_{i}\right\|^{2}
\end{array}\right) \in \operatorname{cone}\left\{\left(\begin{array}{c}
-2 t \\
-\|t\|^{2}
\end{array}\right), t \in T\right\}
$$

with $\sum_{i=1}^{m} \lambda_{i}\left\|t_{i}\right\|^{2}>0$. So, again by $(12), F_{T}\left(0_{n}\right)=\emptyset$. Hence, $F_{T}(s) \neq \emptyset$ implies that $s$ is an extreme point of $\operatorname{conv} T$.

Remark 1 We can also prove the coradiant property of $F_{T}(s) \neq \emptyset$ with very elementary tools, based on the properties of the (not necessarily Euclidean) norm. This is simpler than the proof of [54, Theorem 22]. Let $x \in F_{T}(s)$, take any $\lambda \geq 1$, and put $z:=s+\lambda(x-s)$. Then, for any $t \in T$,

$$
\begin{aligned}
\|z-t\| & =\|s+\lambda(x-s)-t\| \\
& =\|(\lambda-1)(x-s)+(x-t)\| \\
& \leq(\lambda-1)\|(x-s)\|+\|x-t\| \\
& \leq \lambda\|x-s\| \\
& =\|z-s\| .
\end{aligned}
$$

Hence $z \in F_{T}(s)$. 
Statement (ii) was proven in [54] geometrically. The necessary condition for $F_{T}(s) \neq \emptyset$ in Proposition 1(ii) is not sufficient when $T$ is infinite. Indeed, according to [54, Theorem 22], this condition is also sufficient for $F_{T}(s) \neq \emptyset$ when one replaces the set of extreme points of $\operatorname{conv} T$ by a particular class of exposed points of $\operatorname{cl} \operatorname{conv} T$ called boundedly exposed.

We now analyze the existence of cells belonging to the special types of closed convex sets considered in Corollary 5 . To do this we must replace in the latter result, firstly, $S$ by $V_{T}(s)$, and $K(S)$ by $K\left(V_{T}(s)\right)$, and, secondly, $S$ by $F_{T}(s)$, and $K(S)$ by $K\left(F_{T}(s)\right)$. However, there is a substantial difference between both types of cells regarding their possible membership to the families of closed convex sets involved in Corollary 5 (e.g., $V_{T}(s)=\{s\}$ if and only if $K\left(V_{T}(s)\right)$ is a halfspace while $F_{T}(s) \neq\{s\}$ in any case).

Proposition 2 (Existence of special types of cells) Given $n \in \mathbb{N}$, it holds:

(i) For each of the 11 families of sets characterized at Corollary 5 there exist a set $T \subset \mathbb{R}^{n}$ and an element $s \in T$ such that $V_{T}(s)$ belongs to that particular family of sets.

(ii) For each of the three families of sets described at statements (ii), (iv) and (viii) of Corollary 5, but not to those described at the remaining statements, there exist a set $T \subset \mathbb{R}^{n}$ and an element $s \in T$ such that $F_{T}(s)$ belongs to that particular family of sets.

Proof. (i) According to [56, Theorem 2] (whose proof is also based on Theorem 2), given an arbitrary $s \in \mathbb{R}^{n}$ and any closed convex set $C$ such that $s \in C$, there exists a set $T \subset \mathbb{R}^{n}$ such that $C=V_{T}(s)$. So, the 11 cases considered in Corollary 5 are possible.

(ii) We shall use the following characterization of the farthest Voronoi cells [54, Theorem 29]: for any nonempty set $C \varsubsetneqq \mathbb{R}^{n}$, one has $C=F_{T}(s)$ for some $T \subset \mathbb{R}^{n}$ containing $s$ if and only if it is convex, closed, coradiant with respect to $s$, and does not contain $s$.

Since any $F_{T}(s) \neq \emptyset$ is coradiant w.r.t. $s, F_{T}(s)$ is unbounded and so the cases (i), (v), (vi), (ix) and (xi) of Corollary 5 cannot occur.

Since the class of sets described in cases (iii) and (x) of Corollary 5 are contained in that of case (vii), we can preclude the three cases showing, by contradiction, that a farthest Voronoi cell cannot be decomposed as the sum of a compact convex set with a linear subspace. We may assume w.l.o.g. that $s=0_{n}$ does not belong to the given closed convex set $C=D+L$, where $D$ is a compact convex set and $L$ is a linear subspace, with $C$ coradiant w.r.t. $0_{n}$, i.e., $C \subset 0^{+} C$. Then, $D+L=C \subset 0^{+} C=L$, which implies $D \subset L$, so that $0_{n} \in D+L=C$ (contradiction). So, a farthest Voronoi cell cannot belong to the families of sets corresponding to cases (iii), (vii) and (x) of Corollary 5 .

For the remaining cases of Corollary 5 we must provide the corresponding examples. Since any polyhedron is Motzkin decomposable while the full dimensionality is an independent property, it will be sufficient to give a unique example of a full dimensional polyhedron which is farthest Voronoi 
cell. In fact, it is easy to see that $C=\mathbb{R}_{+}^{n}+\{(1,1, \ldots, 1)\}$ is a full dimensional polyhedron such that $C \subset 0^{+} C$, i.e., $C$ is coradiant w.r.t. $0_{n} \notin C$. This completes the proof.

Observe that Lemma 1 can be interpreted as providing characterizations of closed convex coradiant sets and cones to be aggregated at the list of Corollary 5. Example 13 in [54] shows the existence of $T \subset \mathbb{R}^{n}$ and elements $s, s^{\prime} \in \mathbb{R}^{n}$ such that $F_{T}(s)$ is a non-singleton closed convex cone with apex $s^{\prime}$ (so, coradiant w.r.t. $s^{\prime}$ too).

The detection of the boundedness (i.e., compactness), the polyhedrality, the conicity, and the quasipolyhedrality of the cells has deserved particular attention of the previous works on Voronoi cells of infinite sets. The next lemma characterizes the recession cones of both types of Voronoi cells. The recession cone of $V_{T}(s)$ was not explicitly given in [56] while the expression of the recession cone of $F_{T}(s)$ appeared at [54, Proposition 17], but the proof below is much simpler.

Lemma 2 (Recession cones of cells) For $T \subset \mathbb{R}^{n}$ and $s \in T$, it holds that

$$
0^{+} V_{T}(s)=N_{\operatorname{conv} T}(s)
$$

and,

$$
0^{+} F_{T}(s)=-N_{\operatorname{conv} T}(s),
$$

whenever $F_{T}(s) \neq \emptyset$.

Proof. From the linear representations (10) and (11) it follows immediately that

$$
0^{+} V_{T}(s)=\left\{x \in \mathbb{R}^{n}:(t-s)^{\prime} x \leq 0, t \in T\right\}=N_{\operatorname{conv} T}(s)
$$

and

$$
\begin{aligned}
0^{+} F_{T}(s) & =\left\{x \in \mathbb{R}^{n}:-(t-s)^{\prime} x \leq 0, t \in T\right\} \\
& =\left\{-x \in \mathbb{R}^{n}:(t-s)^{\prime} x \leq 0, t \in T\right\} \\
& =-N_{\operatorname{conv} T}(s) .
\end{aligned}
$$

Proposition 3 (Conicity of cells) For $T \subset \mathbb{R}^{n}$ and $s \in T$, the following statements hold:

(i) If $T$ is convex, then $V_{T}(s)$ is a cone with apex $s$.

(ii) If $V_{T}(s)$ is a cone with apex $s$, then $V_{T}(s)=s+N_{\operatorname{conv} T}(s)$.

(iii) $F_{T}(s)$ cannot be a cone with apex $s$.

Proof. (i) comes from [56, Proposition 18]. Observe that $V_{T}(s)=\{s\}$ whenever $s \in \operatorname{int} T$, which is a translate of the trivial cone $\left\{0_{n}\right\}$.

(ii) $V_{T}(s)-s$ is a closed convex cone if and only if $V_{T}(s)-s=0^{+}\left(V_{T}(s)-s\right)=$ $0^{+} V_{T}(s)$, with $0^{+} V_{T}(s)=N_{\operatorname{conv} T}(s)$ by Lemma 2 . 
Proposition 4 (Boundedness of cells) For $T \subset \mathbb{R}^{n}$ and $s \in T$, the following statements hold:

(i) $V_{T}(s)$ is bounded $\Longleftrightarrow s \in \operatorname{int} \operatorname{conv} T$,

(ii) $V_{T}(s)$ is unbounded $\Longleftrightarrow s \in \operatorname{bd} \operatorname{conv} T$,

(iii) $F_{T}(s)$ is either empty or unbounded.

Proof. (i) It follows from Corollary 3 that $V_{T}(s)$ is bounded if and only if $0^{+} V_{T}(s)=\left\{0_{n}\right\}$, and, in view of Lemma 2 , this is equivalent to $N_{\operatorname{conv} T}(s)=$ $\left\{0_{n}\right\}$ which is also equivalent to $s \in \operatorname{int} \operatorname{conv} T$.

(ii) It is immediate from (i) because $s \in T$.

(iii) From Proposition $1\left(\right.$ ii),$F_{T}(s)$ is either empty or coradiant w.r.t. $s$ (and so unbounded).

Statement (iii) above also follows from Proposition 1(ii): if $F_{T}(s) \neq$ $\emptyset$, then $s$ is an extreme point of $\operatorname{conv} T$ and then $\left\{0_{n}\right\} \varsubsetneqq-N_{\operatorname{conv} T}(s)=$ $0^{+} F_{T}(s)$, which gives the unboundedness of $F_{T}(s)$.

From Proposition 4(i) and Proposition 1(ii) one concludes that $V_{T}(s)$ bounded entails $F_{T}(s)=\emptyset$. The converse is true when bd conv $T$ is the set of boundedly exposed points of $\operatorname{cl} \operatorname{conv} T$, as it happens when $T$ is an ellipsoid (in particular, an Euclidean closed ball).

We now give a second formula for $\operatorname{dim} F_{T}(s)$ which is also based on Lemma 2, together with the next lemma, which was shown in [54] using convex analysis tools.

Lemma 3 For $T \subset \mathbb{R}^{n}$ and $s \in T$, one has

$$
F_{T}(s) \subsetneq s-N_{\operatorname{conv} T}(s) .
$$

Proof. From Proposition $1 F_{T}(s)$ is coradiant w.r.t. $s$. Then,

$$
F_{T}(s)-s \subset 0^{+} F_{T}(s) .
$$

Since $0^{+} F_{T}(s)=-N_{\operatorname{conv} T}(s)$ from Lemma 2 , the inclusion (14) follows. The inclusion in (15) is strict since $F_{T}(s)$ cannot be a cone with apex $s$.

Proposition 5 (Dimension of cells via normal cones) If $T \subset \mathbb{R}^{n}$ and $s \in T$ are such that $F_{T}(s) \neq \emptyset$, then

$$
\operatorname{dim} F_{T}(s)=\operatorname{dim} N_{\operatorname{conv} T}(s) .
$$

Proof. Take $x \in F_{T}(s)$. From Lemma 2 and Lemma 3, we have

$$
\begin{aligned}
x-N_{\operatorname{conv} T}(s) & \subset F_{T}(s)-N_{\operatorname{conv} T}(s) \\
& =F_{T}(s)+0^{+} F_{T}(s) \\
& =F_{T}(s) \\
& \subset s-N_{\operatorname{conv} T}(s) .
\end{aligned}
$$

So, $\operatorname{dim} F_{T}(s)=\operatorname{dim} N_{\text {conv } T}(s)$.

The following necessary condition for the cells to be polyhedral, which subsumes [56, Proposition 8], admits a conjoint proof. 
Proposition 6 (Polyhedrality of cells) For $T \subset \mathbb{R}^{n}$ and $s \in T$, the following statements hold:

(i) If $T$ is finite, then $V_{T}(s)$ and $F_{T}(s)$ are polyhedra.

(ii) When either $V_{T}(s)$ or $F_{T}(s)$ is a polyhedron, then clcone $(T-s)$ is polyhedral.

Proof. (i) follows from (10) and (11).

(ii) We can assume w.l.o.g. that $s=0_{n}$. The assumption implies that either $K\left(V_{T}\left(0_{n}\right)\right)$ or $K\left(F_{T}\left(0_{n}\right)\right)$ is finitely generated, i.e., there exist vectors $\left(a_{i}, b_{i}\right) \in \mathbb{R}^{n+1}, i=1, \ldots, m$, such that

$$
\operatorname{clcone}\left\{ \pm\left(\begin{array}{c}
2 t \\
\|t\|^{2}
\end{array}\right), t \in T ;\left(\begin{array}{c}
0_{n} \\
1
\end{array}\right)\right\}=\operatorname{cone}\left\{\left(\begin{array}{c}
a_{i} \\
b_{i}
\end{array}\right), i=1, \ldots, m\right\}
$$

where the sign of $\left(2 t,\|t\|^{2}\right)$ is positive (negative, respectively) when $V_{T}(s)$ $\left(F_{T}(s)\right)$ is a polyhedron. It is easy to see that in the first case,

$$
\text { cl cone } T=\text { cone }\left\{a_{i}, i=1, \ldots, m\right\} .
$$

and in the second case,

$$
\text { cl cone } T=\text { cone }\left\{-a_{i}, i=1, \ldots, m\right\}
$$

which proves that cl cone $T$ is finitely generated and so polyhedral in either case.

The next example shows that the converse of Proposition 6(ii) does not hold.

Example 1 Let $s=0_{2}$ and $T \backslash\{s\}=\{1\} \times \mathbb{R}$. Then, cl cone $T=$ cone $\{(1,0), \pm(0,1)\}$ is polyhedral. However, since $s$ is not even extreme point of $\operatorname{cl} \operatorname{conv} T=$ $[0,1] \times \mathbb{R}$, it holds that $F_{T}(s)=\emptyset$. Observe that $s \in \operatorname{bd} \operatorname{conv} T$, so that $V_{T}(s)$ is unbounded. In fact, according to (10),

$$
V_{T}(s)=\left\{x \in \mathbb{R}^{2}: 2 x_{1}+2 u x_{2} \leq u^{2}+1, u \in \mathbb{R}\right\},
$$

which coincides with the convex hull of the envelope to the bunch of straight lines $2 x_{1}+2 u x_{2}=u^{2}+1, u \in \mathbb{R}$, i.e.,

$$
V_{T}(s)=\left\{x \in \mathbb{R}^{2}: 2 x_{1}+x_{2}^{2} \leq 1\right\},
$$

whose boundary is the parabola $x_{2}^{2}=1-2 x_{1}$. Thus, neither $V_{T}(s)$ nor $F_{T}(s)$ is polyhedron.

The application of Theorem 3 to both types of cells requires the fulfillment of the LFM qualification condition by their respective linear representations. Observe that the closedness conditions (3) and (2) are sufficient conditions for the systems in (10) and (11) to be LFM. 
Proposition 7 (Locally Farkas-Minkowski property) If $T$ is closed and $s$ is an isolated point of $T$, then the systems in (10) and (11), provided that $F_{T}(s) \neq \emptyset$, are LFM. In that case,

$$
n=\operatorname{dim} V_{T}(s)+\operatorname{dim} \operatorname{span}\left(T_{\bar{V}}^{\bar{z}}-s\right)=\operatorname{dim} F_{T}(s)+\operatorname{dim} \operatorname{span}\left(T_{\bar{F}}^{\bar{z}}-s\right),
$$

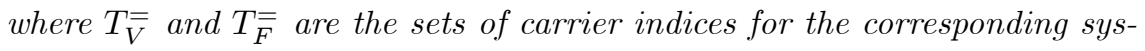
tems.

Proof. Assume that $T$ is closed and $s=0_{n}$ is an isolated point of $T$.

(i) Under the assumptions on $T$ and $s=0_{n}$, cone $\left\{\left(\begin{array}{c}2 t \\ \|t\|^{2}\end{array}\right), t \in T ;\left(\begin{array}{c}0_{n} \\ 1\end{array}\right)\right\}$ is closed [56, Prop. 15(ii)], which entails that (10) is LFM.

(ii) Now, $T$ closed and $F_{T}(s) \neq \emptyset$ give that $T$ is compact by Proposition 1(ii). Since $s$ is an isolated point of $T, T \backslash\left\{0_{n}\right\}$ is compact too, as well as the set

$$
X:=\left\{\frac{1}{\|t\|^{2}}\left(\begin{array}{c}
-2 t \\
-\|t\|^{2}
\end{array}\right), t \in T \backslash\left\{0_{n}\right\}\right\},
$$

by a continuity argument. It is easy to see that $0_{n+1} \notin \operatorname{conv} X$. So, $\mathbb{R}_{+} \operatorname{conv} X$ is closed as well. Furthermore, from $F_{T}(s) \neq \emptyset$ Theorem 1 entails that

$$
\left(\begin{array}{c}
0_{n} \\
-1
\end{array}\right) \notin \text { cone }\left\{\left(\begin{array}{c}
-2 t \\
-\|t\|^{2}
\end{array}\right), t \in T \backslash\left\{0_{n}\right\}\right\}=\mathbb{R}_{+} \operatorname{conv} X .
$$

Finally,

$$
\text { cone }\left\{\left(\begin{array}{c}
-2 t \\
-\|t\|^{2}
\end{array}\right), t \in T \backslash\left\{0_{n}\right\} ;\left(\begin{array}{c}
0_{n} \\
1
\end{array}\right)\right\}=\mathbb{R}_{+} \operatorname{conv} X+\operatorname{cone}\left\{\left(\begin{array}{c}
0_{n} \\
1
\end{array}\right)\right\}
$$

is closed by [69, Corollary 9.1.3]. Then the system (11) is LFM.

The formulas for $\operatorname{dim} V_{T}(s)$ and $\operatorname{dim} V_{F}(s)$ hold from Theorem 3(i).

The application of Theorem 3 to Example 1 is as follows. Since $s=$ $(0,0)$ is a strict solution of the system $\sigma=\left\{2 x_{1}+2 u x_{2} \leq u^{2}+1, u \in \mathbb{R}\right\}$, there is no carrier index, which gives that $\operatorname{dim} V_{T}(s)=2$ and int $V_{T}(s)=$ $\left\{x \in \mathbb{R}^{2}: 2 x_{1}+2 u x_{2}<u^{2}+1, u \in \mathbb{R}\right\}$ is the set of Slater points of $\sigma$.

Regarding the facial structure of the cells, to apply Theorem 4 or Corollary 6 , one needs either the analyticity or the fulfillment or the LOP qualification of their linear representations given by (10) and (11).

One has analytic representations of both types of cells when $T$ is the image of some compact interval $I \subset \mathbb{R}$ by some (componentwise) analytic function $g: I \rightarrow \mathbb{R}^{n}$ and $s=g\left(y_{0}\right)$ for some $y_{0} \in I$. In fact, (10) and (11) are equivalent to

$$
\left\{2(g(y)-s)^{\prime} x \leq\|g(y)\|^{2}-\|s\|^{2}, y \in I\right\}
$$

and to

$$
\left\{-2(g(y)-s)^{\prime} x \leq\|s\|^{2}-\|g(y)\|^{2}, y \in I\right\},
$$


respectively. So, the set $L(x)$ involved in Theorem 4 and Corollary 6 is the same for (20) and for (21). In the non-trivial case that the slack function at $x$, the analytic function $z(y):= \pm\left(2(g(y)-s)^{\prime} x-\|g(y)\|^{2}+\|s\|^{2}\right)$, is not identically null on $I$, to identify $L(x)$ one must compute all zeros of $z$ on $I$ and, for any zero, say $\bar{y}$, whose order of multiplicity we denote by $d(\bar{y})+1$, calculate the successive derivatives $\frac{d z}{d y}(\bar{y}), \ldots, \frac{d^{(d(\bar{y}))} z}{d y^{(d(\bar{y}))}}(\bar{y})$.

Regarding the LOP data qualification, [56, Lemma 21] shows that it is satisfied by (10) whenever $T$ is discrete, in the sense that it has no accumulation point, which allows to assert that $V_{T}(s)$ is a quasipolyhedron. Unfortunately, we cannot provide a farthest counterpart for this result as $F_{T}(s) \neq \emptyset$ implies that $T$ is bounded, and, since the only discrete bounded sets in $\mathbb{R}^{n}$ are the finite ones, $F_{T}(s)$ turns out to be a polyhedron.

\section{Uncertain Voronoi cells}

In many practical applications of Voronoi cells, the position of the generator is accurate (or deterministic) while the positions of some other sites are approximately known (uncertain) due to measurement errors, prediction errors, or to the presence of random factors. For the sake of simplicity we consider in this section finite sets of sites where the generator $s$ is deterministic while the remaining $m$ sites are uncertain, with nominal positions $\bar{t}_{1}, . ., \bar{t}_{m} \in \mathbb{R}^{n}$. We denote by $\bar{T}=\left\{\bar{t}_{0}:=s, \bar{t}_{1}, . ., \bar{t}_{m}\right\}$ the nominal set of sites and by $T=\left\{t_{0}:=s, t_{1}, . ., t_{m}\right\}$ the generic result of perturbing the sites $\bar{t}_{i} \in \bar{T}, i=1, \ldots, m$.

We are interested in guaranteeing the consistency of the robust cells and checking the continuity properties of the multifunctions $\mathcal{V}$ and $\mathcal{F}$ assigning to each $\left(t_{1}, . ., t_{m}\right) \in \mathbb{R}^{n \times m}$ the cells $V_{T}(s)$ and $F_{T}(s)$, respectively. We refer the reader to [57] in order to find an extensive discussion of the stability of a given Voronoi cell $V_{T}(s)$ under different situations of uncertainty, e.g. small perturbations of $s$ in $\mathbb{R}^{n}$, global perturbations of some subset $P \subset T \backslash\{s\}$, and individual perturbations of the elements of the subset $P$.

\subsection{Existence of robust farthest Voronoi cells}

Since the nearest Voronoi cells always satisfy $s \in V_{T}(s)$, for any $s$ and any $T \subset \mathbb{R}^{n}$ such that $s \in T$, arbitrary perturbations of $\bar{t}_{1}, . ., \bar{t}_{m}$ preserve the non-emptiness of the cell. So, this subsection only considers farthest Voronoi cells under perturbations of the sites preserving the generator. Here the nominal system

$$
\bar{\sigma}=\left\{-2\left(\bar{t}_{i}-s\right)^{\prime} x \leq\|s\|^{2}-\left\|\bar{t}_{i}\right\|^{2}, i=1, \ldots, m\right\}
$$


is assumed to have non-empty solution set, $F_{\bar{T}}(s) \neq \emptyset$, where $\bar{T}=\left\{\bar{t}_{0}:=s, \bar{t}_{1}, . ., \bar{t}_{m}\right\}$. Recall that the epigraphical set of $\bar{\sigma}$ is

$$
E(\bar{\sigma})=\operatorname{conv}\left\{\left(\begin{array}{c}
-2\left(\bar{t}_{i}-s\right) \\
\|s\|^{2}-\left\|\bar{t}_{i}\right\|^{2}
\end{array}\right), i=1, \ldots, m\right\}+\mathbb{R}_{+}\left\{\left(\begin{array}{c}
0_{n} \\
1
\end{array}\right)\right\},
$$

which is closed as it is the sum of a polytope with a ray.

Proposition 8 (Positiveness of $d\left(0_{n+1}, E(\bar{\sigma})\right)$ ) If $F_{\bar{T}}(s) \neq \emptyset$, then

$$
d\left(0_{n+1}, E(\bar{\sigma})\right)>0 .
$$

Proof. Assume that $F_{\bar{T}}(s) \neq \emptyset$, and $d\left(0_{n+1}, E(\bar{\sigma})\right)=0$. This means that $0_{n+1} \in E(\bar{\sigma})$ and, so,

$$
0_{n} \in \operatorname{conv}\left\{\left(\bar{t}_{i}-s\right), i=1, \ldots, m\right\},
$$

which yields that $s \in \operatorname{conv}\left\{\bar{t}_{i}, i=1, \ldots, m\right\}$, i.e., $s$ is not an extreme point of conv $\bar{T}$. Proposition 1(ii) provides the contradiction $F_{\bar{T}}(s)=\emptyset$.

Corollary 7 The radius of feasibility for ball uncertainty of non-empty farthest Voronoi cells is always positive. 8.

Proof. It is an straightforward application of Theorem 5 and Proposition

We now assume ball uncertainty sets for the perturbable sites, i.e., uncertainty sets of the form $\mathcal{T}_{i}\left(r_{i}\right)=\bar{t}_{i}+r_{i} B_{n}$, with $r_{i} \geq 0, i=1, \ldots, m$. Obviously, $r_{i}=0$ means that the corresponding site is deterministic (i.e., that only $t_{i}=\bar{t}_{i}$ can occur) as well as the corresponding inequality of $\bar{\sigma}$. Denoting $r:=\left(r_{1}, . ., r_{m}\right)$ and $\mathcal{T}(r):=\{s\} \cup \bigcup_{i=1}^{m} \mathcal{T}_{i}\left(r_{i}\right)$, the robust farthest Voronoi cell is the set of all those points in $\mathbb{R}^{n}$ which are farther from the generator $s$ than to any other conceivable perturbations of $\bar{t}_{1}, . ., \bar{t}_{m}$, $\bigcup_{i=1}^{m} \mathcal{T}_{i}\left(r_{i}\right)$, i.e.,

$$
F_{\mathcal{T}(r)}(s)=\left\{x \in \mathbb{R}^{n}:-2(t-s)^{\prime} x \leq\|s\|^{2}-\|t\|^{2}, t \in \mathcal{T}(r) \backslash\{s\}\right\},
$$

where $t \in \mathcal{T}(r)$ can be replaced by $t \in \bigcup_{i=1}^{m} \mathcal{T}_{i}\left(r_{i}\right)$.

We need an upper bound for the size of the perturbation of the vector $\left(\begin{array}{c}2(t-s) \\ \|t\|^{2}-\|s\|^{2}\end{array}\right)$ when $t$ is perturbed by a vector $\Delta t$. Assume that $\|t\| \leq \mu$ and $\|\Delta t\| \leq 1$. Then

$$
\begin{aligned}
\left\|\left(\begin{array}{c}
2(t+\Delta t-s) \\
\|t+\Delta t\|^{2}-\|s\|^{2}
\end{array}\right)-\left(\begin{array}{c}
2(t-s) \\
\|t\|^{2}-\|s\|^{2}
\end{array}\right)\right\|^{2} & =\left\|\left(\begin{array}{c}
2 \Delta t \\
\|t+\Delta t\|^{2}-\|t\|^{2}
\end{array}\right)\right\|^{2} \\
& =4\|\Delta t\|^{2}+\left(2 t^{\prime} \Delta t+\|\Delta t\|^{2}\right)^{2} \\
& \leq 4\left(1+\mu^{2}\right)\|\Delta t\|^{2}+4 \mu\|\Delta t\|^{3}+\|\Delta t\|^{4} \\
& \leq\left(4 \mu^{2}+4 \mu+5\right)\|\Delta t\|^{2}
\end{aligned}
$$


and, so,

$$
\left\|\left(\begin{array}{c}
2(t+\Delta t-s) \\
\|t+\Delta t\|^{2}-\|s\|^{2}
\end{array}\right)-\left(\begin{array}{c}
2(t-s) \\
\|t\|^{2}-\|s\|^{2}
\end{array}\right)\right\| \leq \sqrt{4 \mu^{2}+4 \mu+5}\|\Delta t\| .
$$

Observe that this bound is sharp by considering any $t$ with $\|t\|=\mu$ and taking $\Delta t=\frac{1}{\mu} t$.

Proposition 9 (Existence of robust farthest Voronoi cell) Assume that $\left\|\bar{t}_{i}\right\| \leq \mu, i=1, \ldots, m$, and let $\mathcal{T}(r)=\{s\} \cup \bigcup_{i}^{m}\left(\bar{t}_{i}+r_{i} B_{n}\right)$, with $r_{i} \in$ $[0,1], i=1, \ldots, m$. Then,

$$
\max _{i=1, \ldots, m} r_{i}<\frac{d\left(0_{n+1}, E(\bar{\sigma})\right)}{\sqrt{4 \mu^{2}+4 \mu+5}} \Longrightarrow F_{\mathcal{T}(r)}(s) \neq \emptyset .
$$

Proof. If $t_{i} \in \bar{t}_{i}+r_{i} B_{n}$, we can write $t_{i}=\bar{t}_{i}+\Delta t_{i}$ with $\left\|\bar{t}_{i}\right\| \leq \mu$ and $\left\|\Delta t_{i}\right\| \leq r_{i} \leq 1$. Then, by $(23)$,

$$
\left(\begin{array}{c}
2\left(t_{i}-s\right) \\
\left\|t_{i}\right\|^{2}-\|s\|^{2}
\end{array}\right) \in\left(\begin{array}{c}
2\left(\bar{t}_{i}-s\right) \\
\left\|\bar{t}_{i}\right\|^{2}-\|s\|^{2}
\end{array}\right)+\sqrt{4 \mu^{2}+4 \mu+5} r_{i} B_{n+1},
$$

with $\sqrt{4 \mu^{2}+4 \mu+5} r_{i}<d\left(0_{n+1}, E(\bar{\sigma})\right)$. The conclusion follows from Theorem 5 and $(7)$.

Actually the bound in (24) is quite conservative, as the following simple example shows. The problem of finding a tight upper bound for the size of the perturbations which preserve the non-emptiness of the farthest Voronoi cells remains open.

Example 2 The following particularly simple case shows that the bound provided by Proposition 9 is very conservative. Let $s=(0,0), \bar{t}=(0,1)$, and $\bar{T}=\{s, \bar{t}\}$ in $\mathbb{R}^{2}$. We can handle this problem geometrically, considering the ball of radius $r$ centered at $\bar{t}$. The generator $s$ is an extreme point of the convex hull of $\mathcal{T}(r)=\{s\} \cup\left(\{(0,1)\}+r B_{2}\right)$ whenever $0 \leq r \leq 1$. Now, $F_{\bar{T}}(s)=\left\{\left(x_{1}, x_{2}\right): 2 x_{2} \geq 1\right\}$, and $F_{\mathcal{T}(1)}(s)=\{(0,1)\}+\mathbb{R}_{+}\{(0,1)\}$, which gives that $F_{\mathcal{T}(r)}(s) \neq \emptyset$ for any $r, 0 \leq r \leq 1$. (See Fig. 1). Moreover, $F_{\mathcal{T}(r)}(s)=\emptyset$ for $r>1$ because then $s$ is not an extreme point of the convex set $\mathcal{T}(r)$. So, $\sup \left\{r: F_{\mathcal{T}(r)}(s) \neq \emptyset\right\}=1$. On the other hand, given that

$$
E(\bar{\sigma})=\left\{\left(\begin{array}{c}
0 \\
-2 \\
-1
\end{array}\right)\right\}+\mathbb{R}_{+}\left\{\left(\begin{array}{l}
0 \\
0 \\
1
\end{array}\right)\right\}
$$

then $d\left(0_{3}, E(\bar{\sigma})\right)$ is obtained as the square root of the optimal value of the quadratic optimization problem

$$
\begin{aligned}
P: \min & f(x)=x_{1}^{2}+x_{2}^{2}+x_{3}^{2} \\
\text { s.t. } & x_{1}=0, \\
& x_{2}=-2, \\
& -x_{3} \leq 1 .
\end{aligned}
$$


The optimal solution of $P$ is $\bar{x}=(0,-2,0)$, with optimal value 4 , so that $\rho(\bar{\sigma})=d\left(0_{3}, E(\bar{\sigma})\right)=2$. For

$$
\mu:=\|(0,1)\|=1
$$

(24) becomes

$$
\max r<\frac{2}{\sqrt{13}} \simeq 0.5547 \Longrightarrow F_{\mathcal{T}(r)}(s) \neq \emptyset .
$$

Since $\sup \left\{r: F_{\mathcal{T}(r)}(s) \neq \emptyset\right\}=1$, we can see that the bound in Proposition 9 is far to be tight.
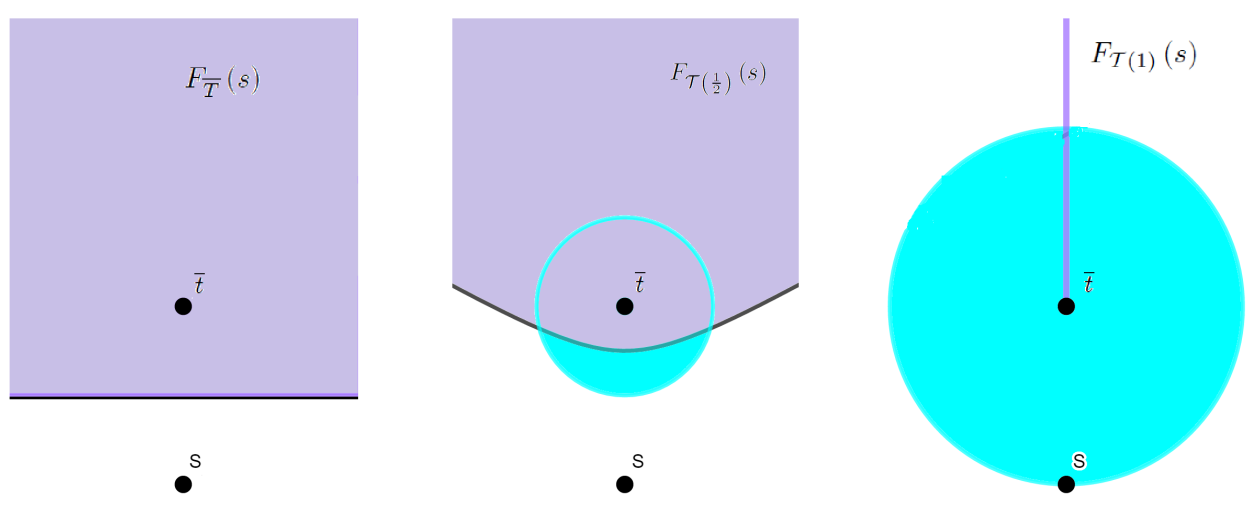

Fig. 1 Robust farthest Voronoi cells

\subsection{Continuity properties of the Voronoi cell mappings}

This final subsection deals with the conditions guaranteeing that $V_{T}(s)$ and $F_{T}(s)$ do not change abruptly when $T$ moves close to a nominal set of sites $\bar{T}=\left\{\bar{t}_{0}:=\bar{s}, \bar{t}_{1}, . ., \bar{t}_{m}\right\}$. We associate with $\bar{T}$ two nominal systems,

$$
\bar{\sigma}_{V}=\left\{2\left(\bar{t}_{i}-s\right)^{\prime} x \leq\left\|\bar{t}_{i}\right\|^{2}-\|s\|^{2}, i=1, \ldots, m\right\}
$$

and

$$
\bar{\sigma}_{F}=\left\{-2\left(\bar{t}_{i}-s\right)^{\prime} x \leq\|s\|^{2}-\left\|\bar{t}_{i}\right\|^{2}, i=1, \ldots, m\right\}
$$

representing $V_{\bar{T}}(s)$ and $F_{\bar{T}}(s)$, respectively, that we can identify with their respective sets of vectors of coefficients,

$$
\left\{\left(\begin{array}{c}
2\left(\bar{t}_{i}-s\right) \\
\left\|\bar{t}_{i}\right\|^{2}-\|s\|^{2}
\end{array}\right), i=1, \ldots, m\right\} \text { and }\left\{\left(\begin{array}{c}
-2\left(\bar{t}_{i}-s\right) \\
\|s\|^{2}-\left\|\bar{t}_{i}\right\|^{2}
\end{array}\right), i=1, \ldots, m\right\} .
$$


We denote by $\Omega$ the class of linear systems $\sigma=\left\{a_{i}^{\prime} x \leq b_{i}, i=1, \ldots, m\right\}$ with the same number of variables and constraints as $\bar{\sigma}_{V}$ and $\bar{\sigma}_{F}$. We identify $\sigma$ with the set $\left\{\left(\begin{array}{l}a_{i} \\ b_{i}\end{array}\right), i=1, \ldots, m\right\} \subset \mathbb{R}^{n+1}$, and consider $\Omega$ endowed with the topology of the uniform convergence. In other words, $\Omega=\mathbb{R}^{(n+1) \times m}$ is equipped with the norm

$$
\|\sigma\|=\max _{i=1, \ldots, m}\left\|\left(\begin{array}{c}
a_{i} \\
b_{i}
\end{array}\right)\right\|
$$

and the corresponding metric

$$
d(\sigma, \widetilde{\sigma})=\|\sigma-\widetilde{\sigma}\|=\max _{i=1, \ldots, m}\left\|\left(\begin{array}{c}
a_{i} \\
b_{i}
\end{array}\right)-\left(\begin{array}{c}
\widetilde{a}_{i} \\
\widetilde{b}_{i}
\end{array}\right)\right\|,
$$

for any $\widetilde{\sigma}=\left\{\widetilde{a}_{i}^{\prime} x \leq \widetilde{b}_{i}, i=1, \ldots, m\right\}$.

We consider small perturbations of $\bar{t}_{1}, . ., \bar{t}_{m}$, say $t_{1}, . ., t_{m}$, which preserve its cardinality. In order to avoid repetitions of sites at the admissible perturbations of $\bar{T}$, we can assume w.l.o.g. that

$$
\left\|t_{i}-\bar{t}_{i}\right\|<\eta, \forall i=0,1, \ldots, m,
$$

where

$$
\eta:=\frac{1}{2} \min \left\{\left\|\bar{t}_{i}-\bar{t}_{j}\right\|: i<j, i, j=1, \ldots, m\right\} .
$$

So, we define another parameter space for the perturbations of the uncertain sites,

$$
\Theta:=\left\{\left(t_{1}, . ., t_{m}\right) \in \mathbb{R}^{n \times m}:\left\|t_{i}-\bar{t}_{i}\right\|<\eta, i=1, \ldots, m\right\},
$$

equipped with the metric

$$
d\left(\left(t_{1}, . ., t_{m}\right),\left(\widetilde{t}_{1}, . ., \widetilde{t}_{m}\right)\right)=\left\|\left(t_{1}, . ., t_{m}\right)-\left(\widetilde{t}_{1}, . ., \widetilde{t}_{m}\right)\right\|:=\max _{i=1, \ldots, m}\left\|t_{i}-\widetilde{t}_{i}\right\|
$$

for any pair $\left(t_{1}, . ., t_{m}\right),\left(\widetilde{t}_{1}, . ., \widetilde{t}_{m}\right) \in \Theta$, and consider the ordinary mappings $h_{V}, h_{F}: \Theta \longrightarrow \Omega$ such that

$$
h_{V}\left(t_{1}, . ., t_{m}\right)=\left\{2\left(t_{i}-s\right)^{\prime} x \leq\left\|t_{i}\right\|^{2}-\|s\|^{2}, i=1, \ldots, m\right\}
$$

and

$$
h_{F}\left(t_{1}, . ., t_{m}\right)=\left\{-2\left(t_{i}-s\right)^{\prime} x \leq\|s\|^{2}-\left\|t_{i}\right\|^{2}, i=1, \ldots, m\right\},
$$

respectively. It is clear that $h_{V}$ and $h_{F}$ are continuous functions.

Let $\mathcal{S}: \Omega \rightrightarrows \mathbb{R}^{n}$ be the solution set mapping introduced in Section 2 . We are interested in the continuity properties of the composite mappings $\mathcal{V}, \mathcal{F}: \Theta \rightrightarrows \mathbb{R}^{n}$ such that $\mathcal{V}=\mathcal{S} \circ h_{V}$ and $\mathcal{F}=\mathcal{S} \circ h_{F}$, i.e.,

$$
\mathcal{V}\left(t_{1}, . ., t_{m}\right)=\left\{x \in \mathbb{R}^{n}: 2\left(t_{i}-s\right)^{\prime} x \leq\left\|t_{i}\right\|^{2}-\|s\|^{2}, i=1, \ldots, m\right\}
$$


and

$$
\mathcal{F}\left(t_{1}, . ., t_{m}\right)=\left\{x \in \mathbb{R}^{n}:-2\left(t_{i}-s\right)^{\prime} x \leq\|s\|^{2}-\left\|t_{i}\right\|^{2}, i=1, \ldots, m\right\},
$$

for each $\left(t_{1}, . ., t_{m}\right) \in \Theta$. Observe that $\operatorname{dom} \mathcal{V}=\Theta$.

The following proposition summarizes the stability properties of the mapping $\mathcal{V}$. Even though parts (ii) and the "if" part in (iii) can be obtained as an application of [57, Theorem 3$]$ with $P=\bar{T} \backslash\{s\}$, we prefer to include direct proofs in the spirit of showing applications of the theory of LSIS. Notice that the property that $\mathcal{V}$ usc at $\left(\bar{t}_{1}, . ., \bar{t}_{m}\right)$ implies that $V_{\bar{T}}(s)$ to be bounded in (iii) is not a direct application of Theorem 7, because of the specific kind of perturbations that are allowed here.

Proposition 10 (Stability of the nearest Voronoi cells) Let $\bar{T}=$ $\left\{\bar{t}_{0}:=s, \bar{t}_{1}, . ., \bar{t}_{m}\right\}$. Then the following statements hold:

(i) $\mathcal{V}$ is closed.

(ii) $\mathcal{V}$ is lsc at $\left(\bar{t}_{1}, . ., \bar{t}_{m}\right)$.

(iii) $\mathcal{V}$ is usc at $\left(\bar{t}_{1}, . ., \bar{t}_{m}\right)$ if and only if $V_{\bar{T}}(s)$ is bounded.

Proof. (i) This follows from the fact that $\mathcal{V}$ is the composition of a continuous function with a closed multifunction.

(ii) It follows in a similar vein as the proof of Theorem 6. Assume that $W$ is any open set in $\mathbb{R}^{n}$ such that $V_{\bar{T}}(s) \cap W \neq \emptyset$ and let $z \in V_{\bar{T}}(s) \cap W$. Observe that $s \in \operatorname{int} V_{\bar{T}}(s)$, because $\bar{T}$ is finite, hence by the accessibility lemma we may consider w.l.o.g. that $z$ is also an interior point of the Voronoi cell $V_{\bar{T}}(s)$. Moreover, we can find $\varepsilon>0$ such that $2\left(\bar{t}_{i}-s\right)^{\prime} z \leq\left\|\bar{t}_{i}\right\|^{2}-$ $\|s\|^{2}-\varepsilon$ for $i=1, \ldots, m$. Now,

$$
\begin{aligned}
2\left(t_{i}-s\right)^{\prime} z & =2\left(\bar{t}_{i}-s\right)^{\prime} z+2\left(t_{i}-\bar{t}_{i}\right)^{\prime} z \\
& \leq\left\|\bar{t}_{i}\right\|^{2}-\|s\|^{2}-\varepsilon+2\left\|t_{i}-\bar{t}_{i}\right\|\|z\| \\
& \leq\left\|t_{i}\right\|^{2}-\|s\|^{2},
\end{aligned}
$$

for any $i=1, \ldots, m$, whenever $\left\|\left(t_{1}, . ., t_{m}\right)-\left(\bar{t}_{1}, . ., \bar{t}_{m}\right)\right\|$ is small enough so that

$$
\left\|t_{i}-\bar{t}_{i}\right\|<\frac{\varepsilon}{4(1+\|z\|)} \text { and }\left\|\bar{t}_{i}\right\|^{2}-\left\|t_{i}\right\|^{2}<\frac{\varepsilon}{2}, i=1, \ldots, m .
$$

Then, defining $T=\left\{t_{0}:=s, t_{1}, . ., t_{m}\right\}$, we have $z \in V_{T}(s) \cap W$, which gives $V_{T}(s) \cap W \neq \emptyset$. Therefore $\mathcal{V}$ is lsc at $\left(\bar{t}_{1}, . ., \bar{t}_{m}\right)$.

(iii) Assume that $V_{\bar{T}}(s)$ is bounded. From Theorem 7, which holds for any type of perturbations of the coefficients in (10), it follows that $\mathcal{V}$ is usc at $\left(\bar{t}_{1}, \ldots, \bar{t}_{m}\right)$. For the converse, assume the upper semi-continuity of $\mathcal{V}$ at $\left(\bar{t}_{1}, . ., \bar{t}_{m}\right)$ and suppose that $V_{\bar{T}}(s)$ is not bounded. Since $V_{\bar{T}}(s) \neq \mathbb{R}^{n}$, we may consider, as in [25, Theorem 3.6], an unbounded sequence $\left\{x_{k}\right\} \subset$ 
bd $V_{\bar{T}}(s)$ with $\left\|x_{k}\right\| \rightarrow \infty$, and take $u=\lim _{k \rightarrow \infty} \frac{-1}{\left\|x_{k}\right\|} x_{k}$, by passing to a subsequence if necessary. Then we have that $\|u\|=1$ and

$$
\lim _{k \rightarrow \infty} u^{\prime} x_{k}=-\infty
$$

Put $M=\max \left\{\left\|\bar{t}_{i}\right\|, i=1, \ldots, m\right\}$ and observe that the set

$$
U=\left\{x \in \operatorname{bd} V_{\bar{T}}(s): u^{\prime} x \leq-M\right\},
$$

is unbounded. Take a sequence $\left\{z_{k}\right\} \subset U$ such that $\left\|z_{k}\right\|+1<\left\|z_{k+1}\right\|$, then, for each $i=1, \ldots, m$, one has

$2\left(\bar{t}_{i}+\frac{1}{k} u-s\right)^{\prime} z_{k}=2\left(\bar{t}_{i}-s\right)^{\prime} z_{k}+\frac{2}{k} u^{\prime} z_{k} \leq\left\|\bar{t}_{i}\right\|^{2}-\|s\|^{2}-\frac{2 M}{k}<\left\|\bar{t}_{i}+\frac{1}{k} u\right\|^{2}-\|s\|^{2}$,

and we may consider an open neighborhood of $z_{k}, U_{k} \subset z_{k}+\frac{1}{k} B_{n}$, such that $U_{k} \subset V_{T_{k}}(s)$ with $T_{k}=\left\{t_{0}:=s, \bar{t}_{1}+\frac{1}{k} u, \ldots, \bar{t}_{m}+\frac{1}{k} u\right\}$. Now, since $z_{k} \in \operatorname{bd} V_{\bar{T}}(s)$ we can take

$$
y_{k} \in U_{k} \backslash V_{\bar{T}}(s) \subset V_{T_{k}}(s) \backslash V_{\bar{T}}(s) .
$$

Hence $\left\{y_{k}\right\}$ is an unbounded sequence with no accumulation point and so $W=\mathbb{R}^{n} \backslash\left\{y_{1}, y_{2}, \ldots\right\}$ is an open set that contains $V_{\bar{T}}(s)$ but $V_{T_{k}}(s) \nsubseteq W$, which is a contradiction with $\mathcal{V}$ being usc at $\left(\bar{t}_{1}, . ., \bar{t}_{m}\right)$. Therefore $V_{\bar{T}}(s)$ is bounded.

Proposition 11 (Stability of the farthest Voronoi cells) Let $\bar{T}=$ $\left\{\bar{t}_{0}:=\bar{s}, \bar{t}_{1}, . ., \bar{t}_{m}\right\}$ be such that $F_{\bar{T}}(s) \neq \emptyset$. The following statements hold: (i) $\mathcal{F}$ is closed.

(ii) If int $F_{\bar{T}}(s) \neq \emptyset$, then $\mathcal{F}$ is lsc at $\left(\bar{t}_{1}, . ., \bar{t}_{m}\right)$.

(iii) $\mathcal{F}$ is not usc at $\left(\bar{t}_{1}, . ., \bar{t}_{m}\right)$.

Proof. (i) and (ii) It is similar to the proof of Proposition 10, just replacing $h_{V}$ by $h_{F}$. In the proof of (ii) consider any $\bar{x} \in \operatorname{int} F_{\bar{T}}(s)$ instead of the site $s$.

(iii) Recall that $F_{\bar{T}}(s)$ is always unbounded when it is not empty by Proposition 4. The proof is similar to the one given in the "only if" part of Proposition 10(iii).

Example 3 Let $\bar{T}=\left\{s, \bar{t}_{1}, \bar{t}_{2}, \bar{t}_{3}\right\}$ with $s=(0,0), \bar{t}_{1}=(2,0), \bar{t}_{2}=(0.2), \bar{t}_{3}=$ $(2,2)$. Since $\left.\left.V_{\bar{T}}(s)=\right]-\infty, 1\right]^{2}$ and $F_{\bar{T}}(s)=\left[1,+\infty\left[^{2}, \mathcal{V}\right.\right.$ and $\mathcal{F}$ are lsc because int $V_{\bar{T}}(s)$ and int $F_{\bar{T}}(s)$ are nonempty. Notice that both cells are unbounded with $0^{+} V_{\bar{T}}(s)$ and $0^{+} F_{\bar{T}}(s)$ being right angles while, replacing the set of sites $\bar{T}=\{(0,0),(2,0),(0,2),(2,2)\}$ by $T_{\varepsilon}=\{(0,0),(2, \varepsilon),(\varepsilon, 2),(2,2)\}$, $\varepsilon>0$ sufficiently small, $0^{+} V_{T_{\varepsilon}}(s)$ and $0^{+} F_{T \varepsilon}(s)$ are angles whose measures increase with $\varepsilon$. Thus, neither $\mathcal{V}$ nor $\mathcal{F}$ are usc at $\left(\bar{t}_{1}, \bar{t}_{2}, \bar{t}_{3}\right)$. 
Remark 2 In the case that $F_{\bar{T}}(s)=\emptyset$, the mapping $\mathcal{F}$ is usc at $\left(\bar{t}_{1}, . ., \bar{t}_{m}\right)$ if and only if $\mathcal{F}$ is also empty in some neighborhood of $\left(\bar{t}_{1}, . ., \bar{t}_{m}\right)$. This is the case, for example, when $s \in \operatorname{int} \operatorname{conv} \bar{T}$. Now, if $s \in \operatorname{bd} \operatorname{conv} \bar{T}$ then $\mathcal{F}$ is not usc at $\left(\bar{t}_{1}, . ., \bar{t}_{m}\right)$. Indeed, for $F_{\bar{T}}(s) \neq \emptyset$ it is just Proposition 11(iii). In the case that $F_{\bar{T}}(s)=\emptyset$, it is always possible to perturb the set $\bar{T} \backslash\{s\}$ in such a manner that $F_{T}(s) \neq \emptyset$. For example one can take a supporting hyperplane of conv $\bar{T}$ at $s$, with an orthogonal vector $w$ of norm 1 such that $\left(\bar{t}_{i}-s\right)^{\prime} w \geq 0, i=1, \ldots, m$. Then, for $\varepsilon>0$ given, define $t_{i}=\bar{t}_{i}+\varepsilon w, i=1, \ldots, m$. In this way, for some $M>0$ large enough we obtain that $z=s+M w \in F_{T}(s)$. Therefore, $\mathcal{F}$ is not usc at $\left(\bar{t}_{1}, . ., \bar{t}_{m}\right)$.

\section{Subdifferentials}

The effective domain, the graph, and the epigraph of $f: \mathbb{R}^{n} \rightarrow \mathbb{R} \cup\{+\infty\}$ are

$$
\begin{gathered}
\operatorname{dom} f:=\left\{x \in \mathbb{R}^{n}: f(x)<+\infty\right\}, \\
\operatorname{gph} f:=\left\{(x, f(x)) \in \mathbb{R}^{n} \times \mathbb{R}: x \in \operatorname{dom} f\right\},
\end{gathered}
$$

and

$$
\text { epi } f:=\left\{(x, \gamma) \in \mathbb{R}^{n} \times \mathbb{R}: x \in \operatorname{dom} f, f(x) \leq \gamma\right\},
$$

respectively, whereas the conjugate function of $f, f^{*}: \mathbb{R}^{n} \rightarrow \mathbb{R} \cup\{+\infty\}$, is defined by

$$
f^{*}(v):=\sup \left\{v^{\prime} x-f(x): x \in \operatorname{dom} f\right\} .
$$

It is well-known that $f^{*}$ is also a proper lower semicontinuous (lsc, in brief) convex function, and that its conjugate, denoted by $f^{* *}$, coincides with $f$ provided that $f$ is a proper lsc convex function.

We denote by $\delta_{X}$ the indicator function of $X \subset \mathbb{R}^{n}\left(\delta_{X}(x)=0\right.$ if $x \in X$ and $\delta_{X}(x)=+\infty$ otherwise) and by $\sigma_{X}$ the support function of $X$

$$
\sigma_{X}(x):=\sup \left\{u^{\prime} x: u \in X\right\} .
$$

Obviously, $\sigma_{X}=\delta_{X}^{*}$. Both functions $\delta_{X}$ and $\sigma_{X}$ are proper, lsc, and convex whenever $X$ is closed and convex. Moreover in this case the reference cone of $X$ is epi $\sigma_{X}$ ([36, Proposition 8]), i.e.,

$$
K(X)=\operatorname{epi} \sigma_{X}
$$

Given $\varepsilon \geq 0$, the $\varepsilon$-subdifferential of $f$ at $a \in \operatorname{dom} f$ is

$$
\partial_{\varepsilon} f(a):=\left\{u \in \mathbb{R}^{n}: f(x) \geq f(a)+u^{\prime}(x-a)-\varepsilon, x \in \operatorname{dom} f\right\} .
$$

The $\varepsilon$-subdifferential is commonly used in convex optimization, but it is also useful to get global optimality conditions for difference-of-convex optimization problems, which includes nonconvex quadratic optimization problems (for example, see [58]). In particular, $\partial_{0} f(a)$ is the so-called Fenchel 
(or convex) subdifferential of $f$ at $a$, usually denoted by $\partial f(a)$. The $\varepsilon$ subdifferentials $\partial_{\varepsilon} f(a)$ are also called approximate subdifferentials when $\varepsilon>0$. Clearly, $\partial_{\varepsilon} f(a)$ is the solution set of the linear inequality system, with unknown $u \in \mathbb{R}^{n}$ and index set $\operatorname{dom} f$,

$$
\left\{(x-a)^{\prime} u \leq f(x)-f(a)+\varepsilon, \quad x \in \operatorname{dom} f\right\} .
$$

Thus, $\partial_{\varepsilon} f(a)$ is a closed convex set. When $\partial_{\varepsilon} f(a) \neq \emptyset$, its recession cone is the solution set of the homogeneous system of (29), i.e.,

$$
0^{+} \partial_{\varepsilon} f(a)=\left\{u \in \mathbb{R}^{n}:(x-a)^{\prime} u \leq 0, \quad x \in \operatorname{dom} f\right\}=N_{\operatorname{dom} f}(a) .
$$

Observe that we also have that $0^{+} \partial_{\varepsilon} f(a)=(\operatorname{cone}(-a+\operatorname{dom} f))^{\circ}$.

It is well known that most relevant information on $\partial_{\varepsilon} f(a)$ is captured by its reference cone $K\left(\partial_{\varepsilon} f(a)\right)$, which in this case, due to (1), is the closure of the characteristic cone of the system (29):

$$
\begin{aligned}
K_{\varepsilon}(f, a) & =\text { cone }\left\{(x-a, f(x)-f(a)+\varepsilon), x \in \operatorname{dom} f ;\left(0_{n}, 1\right)\right\} \\
& =\text { cone }\left\{[\operatorname{gph} f-(a, f(a)-\varepsilon)] \cup\left\{\left(0_{n}, 1\right)\right\}\right\},
\end{aligned}
$$

i.e., $K\left(\partial_{\varepsilon} f(a)\right)=\operatorname{cl} K_{\varepsilon}(f, a)$. Sometimes we write $K(f, a)$ instead of $K_{0}(f, a)$. It is known that $\partial f(a)=\bigcap_{\varepsilon>0} \partial_{\varepsilon} f(a)$. Similarly,

$$
\bigcup_{\varepsilon>0} K_{\varepsilon}(f, a) \subset K(f, a) \subset \operatorname{cl} \bigcup_{\varepsilon>0} K_{\varepsilon}(f, a) .
$$

Given $a \in \operatorname{dom} f$ and $\varepsilon \geq 0$, we will consider the auxiliary function

$$
g(x):=f(x+a)-f(a)+\varepsilon,
$$

which is slightly different to the $h$ function that appears in [69, Section 23]. Obviously, $g: \mathbb{R}^{n} \rightarrow \mathbb{R} \cup\{+\infty\}$ is convex (lsc, proper) if and only if $f$ is convex (lsc, proper, respectively). Moreover, $g\left(0_{n}\right)=\varepsilon$, dom $g=(\operatorname{dom} f)-$ $a, \partial_{\varepsilon} g\left(0_{n}\right)=\partial_{\varepsilon} f(a), \operatorname{gph} g=\operatorname{gph} f-(a, f(a)-\varepsilon)$, and $K_{\varepsilon}\left(g, 0_{n}\right)=$ $K_{\varepsilon}(f, a)$, so that we can assume w.l.o.g. that $f\left(0_{n}\right)=\varepsilon$ in some proofs. It is easy to see that epi $g=$ epi $f-(a, f(a)-\varepsilon)$, so that

$$
K_{\varepsilon}(f, a)=\text { cone epi } g \text {. }
$$

Furthermore,

$$
K_{\varepsilon}(f, a)=\text { cone gph } g, \text { for } \varepsilon>0 .
$$

In the particular case that $f$ is convex, (32) yields $K_{\varepsilon}(f, a)=\mathbb{R}_{+}$epi $g$. Then, taking $\varepsilon=0$ we get

$$
K(f, a)=\text { cone }[\text { epi } f-(a, f(a))]=D(\operatorname{epi} f ;(a, f(a))),
$$

which is the so-called cone of feasible directions of epi $f$ at $(a, f(a))$.

Now, if $f$ is a quasipolyhedral function (i.e., the nonempty intersections of its epigraph with polytopes are polytopes, see [30] and [31]), then $K(f, a)$ is a polyhedral convex cone for any $a \in \operatorname{dom} f$. This is obviously the case when $f$ is polyhedral.

First, we consider the existence of subgradients. The next result is a straightforward consequence of Theorem 1. 


\section{Proposition 12 (Existence of $\varepsilon$-subdifferential (I))}

Let $f: \mathbb{R}^{n} \rightarrow \mathbb{R} \cup\{+\infty\}, a \in \operatorname{dom} f$, and $\varepsilon \geq 0$. Then the following statements are equivalent:

(i) $\partial_{\varepsilon} f(a) \neq \emptyset$.

(ii) $\left(0_{n},-1\right) \notin \operatorname{cl} K_{\varepsilon}(f, a)$.

(iii) $\operatorname{cl} K_{\varepsilon}(f, a) \neq \operatorname{cl}$ cone $(\operatorname{dom} f-a) \times \mathbb{R}$.

From this result one gets well-known results about the subdifferentials of convex functions. First we state a proposition for not necessarily convex functions.

\section{Proposition 13 (Existence of $\varepsilon$-subdifferential (II))}

Let $f: \mathbb{R}^{n} \rightarrow \mathbb{R} \cup\{+\infty\}$ and $a \in \operatorname{dom} f$. If $a \in \operatorname{rint} \operatorname{conv} \operatorname{dom} f$ and $(a, f(a)) \in \operatorname{rbd}$ conv epi $f$, then $\partial_{\varepsilon} f(a) \neq \emptyset$ for all $\varepsilon \geq 0$.

Proof. It is enough to show that $\partial f(a) \neq \emptyset$ because $\partial f(a) \subset \partial_{\varepsilon} f(a)$ for $\varepsilon \geq 0$. Hence, we can assume that $\varepsilon=0$ and, w.l.o.g., that $a=0_{n}$ and $f\left(0_{n}\right)=0$ (by considering the function $g$ in (31) for $\varepsilon=0$ if necessary). Thus $\operatorname{cl} K(f, a)=\operatorname{cl} \mathbb{R}_{+}$convepi $f$, with $0_{n} \in \operatorname{rint} \operatorname{conv} \operatorname{dom} f$ and $\left(0_{n}, 0\right) \in \operatorname{rbd}$ conv epi $f$.

Suppose that $\partial f(a)=\emptyset$. An application of Proposition 12 gives that $\left(0_{n},-1\right) \in \operatorname{cl} K(f, a)=\operatorname{cl} \mathbb{R}_{+}$conv epi $f$. Taking any $M>0$ so that $\left(0_{n}, M\right) \in$ $\operatorname{rint} \mathbb{R}_{+}$conv epi $f$ we obtain, by the accessibility lemma, that $\left(0_{n}, 0\right) \in$ $\operatorname{rint} \mathbb{R}_{+}$conv epi $f$ a contradiction with $\left(0_{n}, 0\right) \in \operatorname{rbd}$ conv epi $f$. Therefore we get the result $\partial f(a) \neq \emptyset$.

Proposition 14 (Existence of $\varepsilon$-subdifferential (III)) Let $f: \mathbb{R}^{n} \rightarrow$ $\mathbb{R} \cup\{+\infty\}$ be a proper convex function and let $a \in \operatorname{rint} \operatorname{dom} f$. Then $\partial_{\varepsilon} f(a) \neq$ $\emptyset$ for all $\varepsilon \geq 0$.

Proof. It follows immediately from Proposition 13 because $\operatorname{dom} f$ and epi $f$ are convex and $(a, f(a)) \in \operatorname{rbd} \operatorname{epi} f$.

\section{Proposition 15 (Existence of $\varepsilon$-subdifferential (IV))}

([59, Theorem XI.1.1.2]) Let $f: \mathbb{R}^{n} \rightarrow \mathbb{R} \cup\{+\infty\}$ be a proper lsc convex function and let $a \in \operatorname{dom} f$. Then $\partial_{\varepsilon} f(a) \neq \emptyset$ for all $\varepsilon>0$.

Proof. Take $\varepsilon>0$ and consider the function $g$ as in (31). Then $g\left(0_{n}\right)=\varepsilon$ and $\operatorname{dom} g=-a+\operatorname{dom} f$. Suppose that $\left(0_{n},-1\right) \in \operatorname{cl} K_{\varepsilon}(f, a)=\operatorname{cl} \mathbb{R}_{+}$epi $g$. Take a convergent sequence $\left\{\lambda_{k}\left(x_{k}, \gamma_{k}\right)\right\}$ in $\mathbb{R}_{+}$epi $g$ with $\lambda_{k} \geq 0, x_{k} \in$ $\operatorname{dom} g, \gamma_{k} \geq g\left(x_{k}\right)$, such that

$$
\lim \lambda_{k}\left(x_{k}, \gamma_{k}\right)=\left(0_{n},-1\right) .
$$

From here $\lambda_{k} x_{k} \rightarrow 0_{n}$ and $\lambda_{k} \gamma_{k} \rightarrow-1$. In particular we get that $g\left(x_{k}\right) \leq$ $\gamma_{k}<0$ for $k$ large enough. Now, if $\liminf \lambda_{k}=0$, we can suppose w.l.o.g. that $\lambda_{k} \in[0,1]$, so $\lambda_{k} x_{k}=\lambda_{k} x_{k}+\left(1-\lambda_{k}\right) 0_{n} \in \operatorname{dom} g$, thus

$$
\begin{aligned}
\varepsilon & =g\left(0_{n}\right) \leq \liminf g\left(\lambda_{k} x_{k}\right) \leq \liminf \lambda_{k} g\left(x_{k}\right)+\left(1-\lambda_{k}\right) g\left(0_{n}\right) \\
& \leq \liminf \lambda_{k} \gamma_{k}+\varepsilon=-1+\varepsilon<\varepsilon,
\end{aligned}
$$


a contradiction. Hence $\lim \inf \lambda_{k}>0$, which yields that $x_{k} \rightarrow 0_{n}$. Then

$$
\varepsilon=g\left(0_{n}\right) \leq \liminf g\left(x_{k}\right) \leq \liminf \gamma_{k} \leq 0,
$$

another contradiction. Therefore, $\left(0_{n},-1\right) \notin \operatorname{cl} K_{\varepsilon}(f, a)$. Finally, an application of Proposition 12 gives the result $\partial_{\varepsilon} f(a) \neq \emptyset$.

Remark 3 The standard proof for Proposition 15 (see [59, Theorem XI.1.1.2]) is simple and it is based on separation of convex sets by hyperplanes. The proof we provide here is focussed in showing that $\left(0_{n},-1\right) \notin \operatorname{cl} K_{\varepsilon}(f, a)$ as an application of Proposition 12, with no further appealing to convex analysis.

Example 4 Consider $f(x)=-\sqrt{1-x^{2}}$ when $x \in[-1,1]$ and $f(x)=+\infty$ otherwise, and take $a=1$, to get $\partial f(a)=\emptyset$. Hence the condition $a \in$ rint dom $f$ is not superfluous in Proposition 14, and we cannot replace $\varepsilon>0$ just by $\varepsilon \geq 0$ in Proposition 15 .

Now we consider the inverse problem consisting of associating with a given closed convex set $F$ a proper lsc convex function $f$ such that $\partial_{\varepsilon} f(a)=$ F.

Proposition 16 (Inverse problem for $\varepsilon$-subdifferential (I)) [59, $E x$ ample XI.1.2.5] For any nonempty closed convex set $F$ and for any $a \in \mathbb{R}^{n}$, $\partial f(a)=F$ whenever $f(x)=\sigma_{F}(x-a)$ for all $x \in \mathbb{R}^{n}$.

Proof. Observe that $\partial f(a)=\left\{u \in \mathbb{R}^{n}: u^{\prime} x \leq \sigma_{F}(x) ; x \in \operatorname{dom} \sigma_{F}\right\}=$ $\partial \sigma_{F}(0)$. Then both sets have the same reference cone,

$$
K(\partial f(a))=K\left(\partial \sigma_{F}\left(0_{n}\right)\right)=\operatorname{cl} K\left(\sigma_{F}, 0_{n}\right)=\operatorname{clepi} \sigma_{F}=\operatorname{epi} \sigma_{F},
$$

since the epigraph of $\sigma_{F}$ is a closed convex cone. Thus, by (28), $K(\partial f(a))=$ epi $\sigma_{F}=K(F)$, which yields $\partial f(a)=F$.

Proposition 17 (Inverse problem for $\varepsilon$-subdifferential (II)) For any nonempty closed convex set $F, a \in \mathbb{R}^{n}$, and $\varepsilon>0$, there exists a proper lsc convex function $f$ such that $\partial_{\varepsilon} f(a)=F$.

Proof. First assume that $F$ is a full dimensional closed convex set. Let $\bar{x} \in \operatorname{int} F$. Then $0_{n} \in \operatorname{int}(F-\bar{x})$, and so the reference cone $K(F-\bar{x}) \subset$ $\mathbb{R}^{n} \times \mathbb{R}_{++} \cup\left\{0_{n+1}\right\}$. For $\varepsilon>0$, consider the closed and convex set $C=$ $\left\{x \in \mathbb{R}^{n}:(x, \varepsilon) \in K(F-\bar{x})\right\}$. Let $g:=\delta_{C}+\varepsilon$, whose epigraph is epi $g=$ $\left\{(x, \gamma) \in \mathbb{R}^{n+1}: x \in C, \gamma \geq \varepsilon\right\}$. Then we have $K(F-\bar{x})=$ cl coneepi $g$. The function $h=\delta_{C+a}$ satisfies $g(x)=h(x+a)-h(a)+\varepsilon$. We have, by $(32) K\left(\partial_{\varepsilon} h(a)\right)=\operatorname{cl} K_{\varepsilon}(h, a)=$ cl cone epi $g$. Thus $\partial_{\varepsilon} h(a)=F-\bar{x}$.

Let $f(x)=h(x)+\langle\bar{x}, x\rangle$, then, by straightforward calculations, we obtain $\partial_{\varepsilon} f(a)=\partial_{\varepsilon} h(a)+\bar{x}=F$.

Now, for a non full dimensional $F$, we can apply this result to aff $F$, which 
w.l.o.g. we may suppose to be some $\mathbb{R}^{k} \times\left\{0_{n-k}\right\} \subset \mathbb{R}^{n}$ with $a \in \mathbb{R}^{k} \times\left\{0_{n-k}\right\}$. Then, get a proper lsc convex function $h: \mathbb{R}^{k} \times\left\{0_{n-k}\right\} \rightarrow \mathbb{R} \cup\{+\infty\}$ with $\partial_{\varepsilon} h(a)=F$. Finally, extend $h$ as $+\infty$ on the rest of $\mathbb{R}^{n}$ to obtain a proper lsc convex function $f: \mathbb{R}^{n} \rightarrow \mathbb{R} \cup\{+\infty\}$ such that $\partial_{\varepsilon} f(a)=F$.

In the following example we will use the fact that $K(F-\bar{x})=M K(F)$, with $M=\left[\begin{array}{cc}I_{n} & 0_{n} \\ -\bar{x}^{\prime} & 1\end{array}\right]$ to produce the function $f$ such that $\partial_{\varepsilon} f(a)=F$.

Example 5 Take $F=[0,2] \times \mathbb{R}$ and $\bar{x}=a=(1,1)$. Then

$K(F)=$ cone $\{(-1,0,0),(1,0,2)\} ; M=\left[\begin{array}{ccc}1 & 0 & 0 \\ 0 & 1 & 0 \\ -1 & -1 & 1\end{array}\right]$, and so $K(F-\bar{x})=$ cone $\{(-1,0,1),(1,0,2)\}$. Thus, $C=\left[(-\varepsilon, 0),\left(\frac{\varepsilon}{2}, 0\right)\right], h(x)=0$ when $x \in$ $\left[(1-\varepsilon, 1),\left(1+\frac{\varepsilon}{2}, 1\right)\right]$ and $h(x)=+\infty$ otherwise, and $f(x)=x_{1}+x_{2}$ when $x=\left(x_{1}, x_{2}\right) \in\left[(1-\varepsilon, 1),\left(1+\frac{\varepsilon}{2}, 1\right)\right]$ and $f(x)=+\infty$ otherwise.

We finish this section with some geometrical results on $\varepsilon$-subdifferentials. Indeed, from Corollary 5, we can address geometrical properties of $\partial_{\varepsilon} f(a)$ by analyzing the cone $K\left(\partial_{\varepsilon} f(a)\right)=\mathrm{cl} K_{\varepsilon}(f, a)$. We will state only few of them (e.g. Propositions 22 and 23) in order to keep simplicity. Thus, we limit ourselves to discuss some results mostly obtained from other known properties of LSIS theory, such as dimension, interior and compactness of $\partial_{\varepsilon} f(a)$.

The next dimension formula follows from Corollary 4 .

Proposition 18 (Dimension of $\varepsilon$-subdifferentials) Let $f: \mathbb{R}^{n} \rightarrow \mathbb{R} \cup\{+\infty\}$, $\varepsilon \geq 0$, and $a \in \operatorname{dom} f$ be such that $\partial_{\varepsilon} f(a) \neq \emptyset$. Then

$$
\operatorname{dim} \partial_{\varepsilon} f(a)=n-\operatorname{dim} \operatorname{lin} K\left(\partial_{\varepsilon} f(a)\right) .
$$

In particular, $\partial_{\varepsilon} f(a)$ is a singleton if and only if

$$
\operatorname{dim} \operatorname{lin} \operatorname{cl} K_{\varepsilon}(f, a)=n .
$$

Theorem 3 gives important properties about the dimension, the interior, the boundary, and the relative boundary of solution sets of LSIS. However, we need no assure that the defining linear semi-infinite system is LFM. The following lemma is referred for the $\varepsilon$-subdifferential case.

Lemma 4 (Locally Farkas-Minkowski property) Let $f: \mathbb{R}^{n} \rightarrow \mathbb{R} \cup\{+\infty\}$ be a function with compact domain. If $f$ is continuous, and $a \in \operatorname{dom} f$ is such that $\partial f(a) \neq \emptyset$, then the system (29) defining $\partial_{\varepsilon} f(a)$ is LFM for $\varepsilon>0$.

Proof. Under the hypothesis on $f$, the system (29) is continuous. Take any $\bar{u} \in \partial f(a) \neq \emptyset$, then for all $x \in \operatorname{dom} f$ and any $\varepsilon>0$, we have

$$
(x-a)^{\prime} \bar{u} \leq f(x)-f(a)<f(x)-f(a)+\varepsilon .
$$


So $\bar{u}$ is a Slater point of the system (29). Therefore this system is LFM.

Observe that Propositions 13 and 14 are very useful in order to apply the previous lemma.

Proposition 19 (Interior of $\varepsilon$-subdifferentials) Let $f: \mathbb{R}^{n} \rightarrow \mathbb{R} \cup\{+\infty\}$ be a continuous convex function with compact domain. Let $a \in \operatorname{rint} \operatorname{dom} f$. Then for $\varepsilon>0$ it holds:

(i) $\operatorname{dim} \partial_{\varepsilon} f(a)=n$,

(ii) int $\partial_{\varepsilon} f(a)=\left\{u \in \mathbb{R}^{n}:(x-a)^{\prime} u<f(x)-f(a)+\varepsilon, \forall x \in \operatorname{dom} f\right\}$.

In particular,

$$
\partial f(a) \subset \operatorname{int} \partial_{\varepsilon} f(a)
$$

Proof. An application of Lemma 4 and Proposition 14 give that the system (29) is LFM. Theorem 3 completes the proof.

The following lemma will be used in order to show that $\partial_{\varepsilon} f(a)$ is compact for rather general functions $f$ whenever $a \in \operatorname{int}$ conv $\operatorname{dom} f$.

Lemma 5 Let $\emptyset \neq X \subset \mathbb{R}^{n}$. Then, $(\text { cone } X)^{\circ}=\left\{0_{n}\right\}$ if and only if $0_{n} \in$ int conv $X$.

Proof. Assume that $(\text { cone } X)^{\circ}=\left\{0_{n}\right\}$. Thus cl cone $X=\left\{0_{n}\right\}^{\circ}=\mathbb{R}^{n}$. If $0_{n} \notin \operatorname{int} \operatorname{conv} X$, then there exists a a hyperplane $H$ containing $0_{n}$ such that $\operatorname{clconv} X$ is contained in one of the half-spaces determined by $H$. This half-space contains also cone $X$ and its closure clcone $X=\mathbb{R}^{n}$, this is a contradiction. Thus $0_{n} \in \operatorname{int} \operatorname{conv} X$.

Conversely, assume that $0_{n} \in \operatorname{int} \operatorname{conv} X$. Let $\varepsilon>0$ be such that $B\left(0_{n} ; \varepsilon\right) \subset$ conv $X$. Then,

$$
\mathbb{R}^{n}=\text { cone } B\left(0_{n} ; \varepsilon\right) \subset \text { cone conv } X=\text { cone } X .
$$

Therefore, $(\text { cone } X)^{\circ}=\left\{0_{n}\right\}$.

Proposition 20 (Compactness of $\varepsilon$-subdifferentials (I)) Let $f: \mathbb{R}^{n} \rightarrow$ $\mathbb{R} \cup\{+\infty\}, a \in \operatorname{dom} f$, and $\varepsilon \geq 0$ be such that $\partial_{\varepsilon} f(a) \neq \emptyset$. Then, $\partial_{\varepsilon} f(a)$ is compact if and only if $a \in \operatorname{int}$ conv $\operatorname{dom} f$.

Proof. Since $\partial_{\varepsilon} f(a)$ is always closed, $\partial_{\varepsilon} f(a)$ being compact is equivalent to being a bounded set, i.e., from Corollary 3 to $\left\{0_{n}\right\}=0^{+} \partial_{\varepsilon} f(a)=$ $N_{\text {dom } f}(a)=(\text { cone } X)^{\circ}$ for $X=-a+\operatorname{dom} f$. From Lemma 5 this is equivalent to $0_{n} \in \operatorname{int}$ conv $X=-a+\operatorname{int}$ conv dom $f$, which holds if and only if $a \in \operatorname{int}$ conv $\operatorname{dom} f$.

Corollary 8 (Compactness of $\varepsilon$-subdifferentials (II)) Let $f: \mathbb{R}^{n} \rightarrow$ $\mathbb{R} \cup\{+\infty\}$ be a proper lsc convex function and let $a \in \operatorname{dom} f$. Then, for all $\varepsilon>0, \partial_{\varepsilon} f(a)$ is compact if and only if $a \in \operatorname{int} \operatorname{dom} f$. 
Proof. Proposition 15 gives that $\partial_{\varepsilon} f(a) \neq \emptyset$. Hence, Proposition 20 applies.

Proposition 21 (Motzkin decomposition of $\varepsilon$-subdifferentials (I)) Let $f: \mathbb{R}^{n} \longrightarrow \mathbb{R} \cup\{+\infty\}$ be a function, $\varepsilon \geq 0$, and $a \in \operatorname{dom} f$ such that $\partial_{\varepsilon} f(a) \neq \emptyset$. Then $\partial_{\varepsilon} f(a)$ is the sum of a compact convex set and a linear subspace for any $\varepsilon \geq 0$ if and only if $\left(0_{n}, 1\right) \in \operatorname{rint} K\left(\partial_{\varepsilon} f(a)\right)$.

Proof. It is a straightforward application of Corollary 5(vii).

Proposition 22 (Motzkin decomposition of $\varepsilon$-subdifferentials (II)) Let $f: \mathbb{R}^{n} \longrightarrow \mathbb{R} \cup\{+\infty\}$ be a proper convex function. Then, $a \in \operatorname{rint} \operatorname{dom} f$ if and only if $\partial_{\varepsilon} f(a)$ is the sum of a nonempty compact convex set and a linear subspace for any $\varepsilon \geq 0$.

Proof. Let $\varepsilon \geq 0$. First, suppose that $a \in \operatorname{rint} \operatorname{dom} f$. Then, the assumptions on $f$ and Proposition 14 guarantee that $\partial_{\varepsilon} f(a) \neq \emptyset$. On the other hand, $a \in \operatorname{rint} \operatorname{dom} f$ implies that $0_{n} \in \operatorname{rint} \operatorname{dom} g$ where $g$ is the function defined in (31) with $g\left(0_{n}\right)=\varepsilon$. Thus, $\left(0_{n}, 1\right) \in \operatorname{rint}$ cone epi $g=\operatorname{rint} K\left(\partial_{\varepsilon} f(a)\right)$ and, by Proposition 21, $\partial_{\varepsilon} f(a)$ is the sum of a compact convex set with a linear subspace.

Conversely, if $\partial_{\varepsilon} f(a) \neq \emptyset$ is the sum of a compact convex set and a linear subspace for any $\varepsilon \geq 0$, then Proposition 21 gives that $\left(0_{n}, 1\right) \in \operatorname{rint} K\left(\partial_{\varepsilon} f(a)\right)$ $=\operatorname{rint}$ cone epi $g$, which implies that $0_{n} \in \operatorname{rint} \operatorname{dom} g$, equivalently $a \in$ rint $\operatorname{dom} f$.

The particular case $\varepsilon=0$ is [53, Corollary 7].

\section{Proposition 23 (Polyhedrality of $\varepsilon$-subdifferentials)}

Let $f: \mathbb{R}^{n} \longrightarrow \mathbb{R} \cup\{+\infty\}$ be a quasipolyhedral convex function and let $a \in \operatorname{dom} f$. Then $\partial_{\varepsilon} f(a)$ is a nonempty polyhedral convex set for any $\varepsilon \geq 0$.

Proof. If $f$ is a quasipolyhedral convex function, then epi $f-(a, f(a))$ is a polyhedral convex set containing $\left(0_{n}, 0\right)$. Thus, the convex cone generated by epi $f-(a, f(a))$ is polyhedral and so it is closed. Then $K\left(\partial_{\varepsilon} f(a)\right)=$ cone (epi $f-(a, f(a)))$ is polyhedral and $\left(0_{n},-1\right) \notin K\left(\partial_{\varepsilon} f(a)\right)$. Thus $\partial_{\varepsilon} f(a) \neq$ $\emptyset$ and, by Corollary 5(ii), it is a polyhedral convex set.

The facial structure of $\partial_{\varepsilon} f(a)$ can be analyzed using Theorem 4 or Corollary 6 as it was already established in Section 3 regarding the facial structure of Voronoi cells. One needs either the analyticity or the fulfillment or the LOP qualification of a linear representation of $\partial_{\varepsilon} f(a)$. In a similar way as for Voronoi cells, one has an analytic representation of $\partial_{\varepsilon} f(a)$ when $\operatorname{dom} f$ is the image of some compact interval $I \subset \mathbb{R}$ by some (componentwise) analytic function $g: I \rightarrow \mathbb{R}^{n}$. In fact, (29) is equivalent to

$$
\left\{(g(y)-a)^{\prime} u \leq f(g(y))-f(a)+\varepsilon, \quad y \in I\right\}
$$


So, to identify the set $L(u)$ involved in Theorem 4 and Corollary 6 one must consider the slack function at $u, z(y):=(g(y)-a)^{\prime} u-f(g(y))+f(a)-\varepsilon$, $y \in I$. Since $z(y)=-\varepsilon$ when $g(y)=a, z$ is not identically null on $I$. Thus, to describe $L(u)$ one must compute all zeros of $z$ on $I$ and, for any zero, say $\bar{y}$, with order of multiplicity $d(\bar{y})+1$, calculate the successive derivatives $\frac{d z}{d y}(\bar{y}), \ldots, \frac{d^{(d(\bar{y}))} z}{d y^{(d(\bar{y}))}}(\bar{y})$.

Finally, notice that we can also apply Theorems 6 and 7 to analyze the stability of $\partial_{\varepsilon} f(a)$ under perturbations of the function $f$ measured by the pseudometric of the uniform convergence (see, e.g., [62]), in a similar way as we did in Section 4 concerning the Voronoi mappings $\mathcal{V}$ and $\mathcal{F}$. One can obtain stability results for the set-valued mapping $f \longmapsto \partial_{\varepsilon} f(a)$ such as closedness, lower semi-continuity and upper semi-continuity.

\section{Conclusions}

The algebraization of geometry (and all of mathematics) started in the 17th Century with Descartes' Dioptrique (1637), where he reformulated geometric problems related with triangles, until then solved with ruler and compass, in terms of algebraic equations to be solved in radicals. Despite the criticisms of some of his contemporaries, as Jean-Baptiste Morin and Pierre de Fermat, Descartes' algebraic view of mathematics has advanced in many fields along the last four centuries, the paradigm being the algebraic reformulation of geometrical problems involving the zeros of multivariate polynomials, allowing to apply algebraic techniques (in particular, commutative algebra). Even though for many respectable lovers of the constructive geometric methods this phenomenon is nothing else than the result of the fight of "the devil of abstract algebra" against "the angel of geometry" (Hermann Weyl dixit), we think that the algebraization of geometry has practical advantages, as it allows the obtention of new results and the simplification of previous proofs.

This paper pretends to be a modest step forward in the (linear) algebraization of computational geometry and mathematical analysis, trying to illustrate how useful can be the theory of linear semi-infinite systems in the study of Voronoi cells of arbitrary sets and $\varepsilon$-subdifferentials. It can be objected that these simple proofs are possible after obtaining the necessary algebraic tools, but these tools can be used in different settings. For instance, our straightforward proof that any farthest Voronoi cell is coradiant in Proposition 1 is based on the dual characterization of the coradiant sets in Lemma 1, a result which has potential applications in other fields.

However, we do not claim that all known results on Voronoi cells and $\varepsilon$-subdifferentials can be obtained this way. For instance, since no dual characterization of the boundedly exposed points of closed convex sets is available for the moment, the nonempty farthest Voronoi cells have not been characterized here (the proof in [54, Theorem 22] is based on analytic arguments). 
In summary, it would be unrealistic to claim at present that the theories of Voronoi cells and $\varepsilon$-subdifferentials are mere consequence of the existing theory of linear semi-infinite systems. On the contrary, we strongly believe that Voronoi cells of arbitrary sets and $\varepsilon$-subdifferentials will walk a long time on two legs: mathematical analysis and LSIS.

\section{Acknowledgement}

The authors wish to thank the two anonymous referees for their careful reading of the manuscript and for their valuable comments.

This research was partially supported by PGC2018-097960-B-C22 of the Ministerio de Ciencia, Innovación y Universidades (MCIU), the Agencia Estatal de Investigación (AEI), and the European Regional Development Fund (ERDF); by CONICET, Argentina, Res D N ${ }^{\circ} 4198 / 17$; and by Universidad Nacional de Cuyo, Secretaría de Investigación, Internacionales y Posgrado (SIIP), Res. 3922/19-R, Cod.06/D227, Argentina.

\section{References}

1. Anderson, E.J., Goberna, M.A., López, M.A.: Locally polyhedral linear semiinfinite systems. Linear Algebra Appl. 270, 231-253 (1998)

2. Anderson, E.J., Goberna, M.A., López, M.A.: Simplex-like trajectories on quasi-polyhedral sets. Math. Oper. Res. 26, 147-162 (2001)

3. Anderson, E.J., Lewis, A.S.: An extension of the simplex algorithm for semiinfinite linear programming. Math. Programming Series A 44, 247-269 (1989)

4. Bean, N.G., Fackrell, M., Taylor, P.: Characterization of Matrix-Exponential Distributions. Stochastic Models 24, 339-363 (2008)

5. Beer, G., Cánovas, M.J., López, M.A., Parra, J.: A Uniform Approach to Hölder Calmness of Subdifferentials. J. Convex Anal. 27, 167-180 (2020).

6. Ben-Tal, A., El Ghaoui, L., Nemirovski, A.: Robust Optimization. Princeton U.P., Princeton (2009)

7. Brøndsted, A., Rockafellar, R.T.: On the subdifferentiability of convex functions. Proc. Amer. Math. Soc. 16, 605-611 (1965)

8. Brosowski, B.: Parametric Semi-Infinite Optimization. Peter Lang, Frankfurt am Main (1982)

9. Brosowski, B.: Parametric semi-infinite linear programming I. Continuity of the feasible set and the optimal value. Math. Program. Study 21, 18-42 (1984)

10. Cánovas, M.J., Dontchev, A.L., López, M.A., Parra, J.: Metric regularity of semi-infinite constraint systems. Math. Program. 104B, 329-346 (2005)

11. Cánovas, M.J., Gómez-Senent, F.J. Parra, J.: Stability of systems of linear equations and inequalities: distance to ill-posedness and metric regularity. Optimization 56, 1-24 (2007).

12. Cánovas, M.J., Gómez-Senent, F.J., Parra, J.: Regularity modulus of arbitrarily perturbed linear inequality systems. J. Math. Anal. Appl. 343, 315-327 (2008)

13. Cánovas, M.J., López, M.A., Parra, J.: Upper semicontinuity of the feasible set mapping for linear inequality systems. Set-Valued Anal. 10, 361-378 (2002) 
14. Cánovas, M.J., López, M.A., Parra, J., Todorov, M.I.: Stability and wellposedness in linear semi-infinite programming. SIAM J. Optim. 10, 82-89 (1999)

15. Cánovas, M.J., López, M.A., Parra, J., Toledo, F.J.: Distance to ill-posedness and the consistency value of linear semi-infinite inequality systems. Math. Program. 103A, 95-126 (2005)

16. Cánovas, M.J., López, M.A., Parra, J., Toledo, F.J.: Sufficient conditions for total ill-posedness in linear semi-infinite optimization. European J. Oper. Res. 181, 1126-1136 (2007)

17. Cánovas, M.J., López, M.A., Parra, J., Toledo, F.J.: Error bounds for the inverse feasible set mapping in linear semi-infinite optimization via a sensitivity dual approach. Optimization 56, 547-563 (2007)

18. Cánovas, M.J., Mordukhovich, B., López, M.A., Parra, J.: Variational analysis in semi-infinite and infinite programming, I: Stability of linear inequality systems of feasible solutions. SIAM J. Optim. 20, 1504-1526 (2009)

19. Charnes, A., Cooper, W.W., Kortanek, K.O.: Duality, Haar programs, and finite sequence spaces. Proc. Nat. Acad. Sci. USA 48, 783-786 (1962)

20. Charnes, A., Cooper, W.W., Kortanek, K.O.: Duality in semi-infinite programs and some works of Haar and Carathéodory. Management Sci. 9, 209$228(1963)$

21. Charnes, A., Cooper, W.W., Kortanek, K.O.: On representations of semiinfinite programs which have no duality gaps. Management Sci. 12, 113-121 (1965)

22. Chu, Y.C: Generalization of some fundamental theorems on linear inequalities. Acta Math Sinica 16, 25-40 (1966)

23. Combettes, P.L.: The convex feasibility problem in image recovery. In: Hawkes P. (ed.), Advances in Imaging and Electron Physics, vol. 95, pp. 155-270. Academic Press, New York (1996)

24. Coxeter, H.S.M.: Regular Polytopes (3rd ed.). Dove, New York (1973)

25. Daniilidis, A., Goberna, M.A., López, M.A., Lucchetti, R.: Stability in linear optimization under perturbations of the left-hand side coefficients. Set-Valued Var. Anal. 23, 737-758 (2015).

26. Davila, C.E.: Signal denoising via quadratic semi-infinite programming. Conference on Signals, Systems and Computers, 2016-February, 7421203, pp. 612616.

27. Delaunay, B.: Sur la sphère vide: À la mémoire de Georges Voronoi (French). Izv. Akad. Nauk SSSR, Otdelenie Matematicheskih i Estestvennyh Nauk 7, 793-800 (1934)

28. Eckhardt, U.: Theorems on the dimension of convex sets, Linear Algebra Appl. 12, 63-76 (1975)

29. Eckhardt, U.: Representation of convex sets, in Extremal Methods and Systems Analysis (A.V. Fiacco and K.0. Kortanek, eds.), Springer, New York, pp. 374-383 (1980)

30. Fajardo, M.D., López, M.A.: Some results on quasipolyhedral convexity. Top 15, 103-113 (2007)

31. Fajardo, M.D., López, M.A., Puente, R.: Linear representations and quasipolyhedrality of a finite-valued convex function, Optimization 57, 215-237 (2008)

32. Fan, K.: On infinite systems of linear inequalities, J. Math. Anal. Appl. 21, 475-478 (1968) 
33. Fang, S.C., Wu, S.Y., Birbil, S.I.: Solving variational inequalities defined on a domain with infinitely many linear constraints. Comput. Optim. Appl. 37, $67-81(2007)$

34. Ferrer, A., Goberna, M.A., González-Gutiérrez, E., Todorov, M.I.: A comparative study of relaxation algorithms for the linear semi-infinite feasibility problem. Ann Oper. Res. 258, 587-612 (2017)

35. Fischer, T.: Contributions to semi-infinite linear optimization. Meth. Verf. Math. Phys. 27, 175-199 (1983)

36. Goberna, M.A., González, E., Martínez-Legaz, J.E., Todorov, M.I.: Motzkin decomposition of closed convex sets. J. Math. Anal. Appl. 364, 209-221 (2010)

37. Goberna, M.A., Hernández, L., Todorov, M.I.: Separating the solution sets of analytical and polynomial systems. Top 13, 321-329 (2005)

38. Goberna, M.A., Jeyakumar, V., Li, G., Vicente-Pérez, J.: Robust solutions of multiobjective linear semi-infinite programs under constraint data uncertainty. SIAM J. Optim. 24, 1402-1419 (2014)

39. Goberna, M.A., Jeyakumar, V., Li, G., Vicente-Pérez, J.: Robust solutions to multi-objective linear programs with uncertain data. European J. Oper. Res. 242, 730-743 (2015)

40. Goberna, M.A., Jornet, V., Puente, R., Todorov, M.I.: Analytical linear inequality systems and optimization. J. Optim. Theory Appl. 103, 95-119 (1999)

41. Goberna, M.A., Jornet, V., Rodríguez, M.M.L.: On the characterization of some families of closed convex sets, Contributions to Algebra and Geometry 43, 153-169 (2002)

42. Goberna, M.A., Larriqueta, M., Vera de Serio, V.N.: On the stability of the boundary of the feasible set in linear optimization. Set-Valued Analysis 11, 203-223 (2003)

43. Goberna, M.A., Larriqueta, M., Vera de Serio, V.N.: On the stability of the extreme point set in linear optimization. SIAM J. Optim. 15, 1155-1169 (2005)

44. Goberna, M.A., López, M.A.: A theory of linear inequality systems. Linear Algebra Appl. 106, 77-115 (1988)

45. Goberna, M.A., López, M.A.: Topological stability of linear semi-infinite inequality systems. J. Optim. Theory Appl., 89, 227-236 (1996)

46. Goberna, M.A., López, M.A.: Linear Semi-Infinite Optimization. J. Wiley, Chichester (1998)

47. Goberna, M.A., López, M.A.: Recent contributions to linear semi-infinite optimization: An update, Ann. Oper. Res. 271, 237-278 (2018)

48. Goberna, M.A., López, M.A., Pastor, J.T.: Farkas-Minkowski systems in semiinfinite programming. Appl. Math. Optim. 7, 295-308 (1980)

49. Goberna, M.A., López, M.A., Todorov, M.I.: Stability theory for linear inequality systems. SIAM J. Matrix Anal. Appl. 17, 730-743 (1996)

50. Goberna, M.A., López, M.A., Todorov, M.I.: Stability theory for linear inequality systems. II: Upper semicontinuity of the solution set mapping. SIAM J. Optim. 7, 1138-1151 (1997)

51. Goberna, M.A., López, M.A., Todorov, M.I.: On the stability of the feasible set in linear optimization. Set-Valued Anal. 9, 75-99 (2001)

52. Goberna, M.A., López, M.A., Volle, M.: Primal attainment in convex infinite optimization duality. J. Convex Anal. 21, 1043-1064 (2014)

53. Goberna, M.A., Martínez-Legaz, J.E., Todorov, M.I.: On Motzkin decomposable sets and functions. J. Math. Anal. Appl. 372, 525-537 (2010)

54. Goberna, M.A., Martínez-Legaz, J.E., Todorov, M.I.: Farthest Voronoi cells of infinite sets. Linear Algebra Appl. 583, 306-322 (2019) 
55. Goberna, M.A., Martínez-Legaz, J.E., Vera de Serio, V.N.: The Voronoi inverse mapping. Linear Algebra Appl. 504, 248-271 (2016)

56. Goberna, M.A., Rodríguez, M.M.L., Vera de Serio, V.N.: Voronoi cells of arbitrary sets via linear inequality systems. Linear Algebra Appl. 436, 2169$2186(2012)$

57. Goberna, M.A., Vera de Serio, V.N.: On the stability of Voronoi cells, Top 20, 411-425 (2012)

58. Hiriart-Urruty, J.-B.: Conditions for global optimality II. J. Global Optim. 13, 349-367 (1998)

59. Hiriart-Urruty, J.-B., Lemaréchal, C.: Convex Analysis and Minimization Algorithms. I. Fundamentals. Springer, Berlin, (1993)

60. Jaume, D., Puente, R., Representability of convex sets by analytical linear inequality systems. Linear Algebra Appl. 380, 135-150 (2004)

61. Larriqueta, M., Vera de Serio, V.N.: On metric regularity and the boundary of the feasible set in linear optimization. Set-Valued Var. Anal. 22, 1-17 (2014)

62. López, M.A.; Vera de Serio, V. N.: Stability of the feasible set mapping in convex semi-infinite programming. Semi-infinite programming (Alicante, 1999), 101-120, Nonconvex Optim. Appl., 57, Kluwer Acad. Publ., Dordrecht (2001).

63. Moreau, J.-J.: Fonctions sous-différentiables. Note aux C.R. Acad. Sci. Paris 257, 4117-4119 (1963)

64. Prékopa, A., Ninh, A., Alexe, G.: On the relationship between the discrete and continuous bounding moment problems and their numerical solutions. Ann. Oper. Res. 238, 521-575 (2016)

65. Preparata, F.P., Shamos, M.I.: Computational Geometry: An Introduction. Springer, New York (1985)

66. Puente, R.: Cyclic convex bodies and optimization moment problems. Linear Algebra Appl. 426, 596-609 (2007)

67. Puente, R., Vera de Serio, V.N.: Locally Farkas-Minkowski linear semi-infinite systems. Top 7, 103-121 (1999)

68. Rockafellar, R.T.: Convex functions and dual extremum problems. Ph.D. thesis, Harvard University (1963)

69. Rockafellar, R.T.: Convex Analysis. Princeton U.P., Princeton (1970)

70. Voigt, I., Weis, S.: Polyhedral Voronoi cells. Beiträge Algebra Geom. 51, 587$598(2010)$ 